\title{
Linear transport of solar wind fluctuations
}

\author{
Sean Oughton \\ Department of Mathematics, University College London, England \\ William H. Matthaeus \\ Bartol Research Institute, University of Delaware, Newark
}

\begin{abstract}
Numerical solutions for the linear transport of solar wind fluctuations are presented. The model used takes into account the effects of advection, expansion, and wave propagation, as well as the recently illuminated effects of (non-WKB) "mixing" terms. The radial evolution of the fluctuating kinetic and magnetic energies and of the cross helicity is computed, and it is demonstrated that in appropriate limits the solutions converge to the WKB forms. In more general cases, however, where the fluctuations consist of a superposition of various types of turbulence, mixing leads to solutions which differ substantially from those predicted by WKB theory. The degree of mixing shows considerable dependence on the nature of the turbulence, giving rise to varying levels, at $1 \sim \mathrm{AU}$, of the ratio of "inward" and "outward" fluctuation energies and the ratio of kinetic and magnetic fluctuation energies. The transport properties described here may provide a partial explanation for the observed decrease of cross helicity with increasing heliocentric distance in the solar wind.
\end{abstract}

\section{Introduction}

Traditionally, the large-scale evolution of solar wind fluctuations has been investigated using WKB theory [e.g., Parker, 1965; Hollweg, 1973a, b, 1974; Barnes, 1979]. To leading order in this approach the fluctuations are noninteracting (Alfvén) waves which propagate along the solar wind's slowly varying background magnetic field, $\mathbf{B}_{0}$. Propagation is either toward or away from the Sun, respectively referred to as modes of inward and outward type (in what follows we will also use these two terms for nonwave fluctuations, though employing the modifier "type" to emphasize the nonwave nature). The underlying physical picture considers the fluctuations to be almost all of the outward propagating kind, being, to leading order, passive remnants of coronal processes. Some observed features of the evolution, such as the radial dependence of the magnetic fluctuation level in the outer heliosphere, are reasonably well predicted using WKB theory [Roberts et al., 1990]; however, this approach fails to fully account for some other important aspects of the radial evolution. In particular, leading order WKB theory cannot adequately explain (1) the observed level of heating in the solar wind, (2) the dependence of power-spectra slopes on frequency and heliocentric distance, (3) the tendency for the minimum variance direction of the fluctuations to align with $\mathbf{B}_{0}$, and (4) the radial evolution of the

Copyright 1995 by the American Geophysical Union.

Paper number 95JA00628.

0148-0227/95/95JA-00628 $\$ 05.00$ normalized cross helicity and the Alfvén ratio. Furthermore, higher-order corrections are, in general, unable to significantly reduce the discrepancy [e.g., Hollweg, 1990]. Thus, an extended or new approach is required.

Zhou and Matthaeus [1989, 1990a, b, c] have developed a distinct but WKB-related theory for the transport of MHD scale turbulence in a weakly inhomogeneous background plasma, and applied it to the solar wind. This newer theory, based upon a multiple-scales analysis, can be shown to be more general than that of WKB since it remains applicable in cases where the latter is no longer valid [Zhou and Matthaeus, 1990b; Matthaeus et al., 1992; Matthaeus et al., 1994b]. Moreover, the new approach permits, with a single transport model, treatment of fluctuations with arbitrary rotational symmetry, as well as local dynamical interactions among the fluctuations which can be represented by turbulence models. Non-WKB transport models have also been developed independently by the Lindau group [Marsch and Tu, 1989, 1990, 1993; Tu and Marsch, $1990,1992,1993]$. These models are very similar to and in certain cases formally identical to the models presented by Zhou and Matthaeus and here. However, certain technical differences in the derivations of the models lead to potentially important differences in the interpretations of the two models in some applications. We will briefly attempt to clarify some of these points in subsequent sections.

In this paper we further extend the model's application to the solar wind, focusing specifically on the linear transport of inertial range fluctuations. (For solar wind turbulence the inertial range is usually associated with 
the set of scales much less than the fluctuation correlation length and much greater than the ion gyroradius, for which nonlinear interactions are expected to be important, and which are often observed to have power law energy spectra.) This concentration on the linear transport is pursued for several reasons. First, it is the simplest case to examine. Second, if the fluctuations are assumed to represent locally fully-developed steady MHD turbulence, some of the nonlinear terms have no net effect within the inertial range, or alternatively have effects for which no good model yet exists [Zhou and Matthaeus, 1990c]. Third, it focuses attention on the sometimes leading order influence of mixing. The linear versions of the transport model are particularly important for examining whether the inertial range might be substantially influenced by mixing and other transport effects, such as nonequipartition of kinetic and magnetic energies. This is because turbulence geometry has a strong influence on the transport, and linear transport theory permits arbitrary admixtures of fluctuations with differing symmetries to be treated by simple superposition. Thus, in our preliminary report on numerical solutions of linear solar wind transport [Oughton and Matthaeus, 1992], we discussed superpositions of several types of turbulence, namely that with slab, two-dimensional (2D), or isotropic symmetry. These composite models are motivated in part by the suggestion of Matthaeus et al. [1990] that the axisymmetric magnetic autocorrelation function, inferred from ISEE interplanetary observations at $1 \mathrm{AU}$, can be interpreted as a two-component (slab and 2D) distribution of magnetic fluctuations. Multiple component transport, with mixing effects included, has also been discussed by Tu and Marsch [1992, 1993] and Marsch and Tu [1993].

An important feature of non-WKB approaches (both ours and the Lindau group's) is that they support completely arbitrary ratios of energies in the inward- and outward-type fluctuations. Indeed, separate transport equations for both these (and other) quantities arise quite naturally in the models. This is to be contrasted with WKB theory [e.g., Parker, 1965; Hollweg, 1973b, 1974] and the earlier models of Tu and colleagues [ $T u$ et al., 1984; Tu, 1988], which only considered the case when this ratio is small, and often further restricted to be a constant independent of heliocentric distance (the work of Heinemann and Olbert [1980] is an important exception in this regard). On the other hand, observations [e.g., Bavassano et al., 1982a, b; Roberts et al., $1987 \mathrm{a}, \mathrm{b}]$ show that while such an approximation is sometimes valid in the solar wind, there are also many instances when it is not.

The rest of the paper is structured as follows. In section 2 we introduce the notation and briefly emphasize some important features of the model, particularly in relation to other models. Section 3 discusses the structure of the mixing tensors, and also their role in the transport equations. Numerical results are presented in section 4 as is a comparison with observational data. The final section is a discussion one.

\section{The Model}

A complete derivation of the model, along with an extensive discussion of its relative merits and limitations, has been given previously [Zhou and Matthaeus, 1990a, b, c; Matthaeus and Zhou, 1989], so that we do not repeat the details here. Note, however, that whereas in these earlier papers solutions of the transport equations were discussed primarily in terms of reduced (1-D) spectra [e.g., Batchelor, 1970], here we work with omnidirectional wavenumber spectra. The reasons for this switch will be discussed below. Closely related models were derived independently by the Lindau group [Marsch and Tu, 1989, 1993; Tu and Marsch, 1990, 1993].

The objective is to derive (large-scale) spatial transport equations for various (small-scale) spectral tensors characterizing the fluctuations. This can be achieved by assuming that the characteristic length scales for the fluctuations are much smaller than those for the mean fields, permitting a multiple-scales analysis to be performed. Eventually [Zhou and Matthaeus, 1990a], we obtain the leading order, linear, transport equations for the four spectra of interest here:

$$
\begin{aligned}
\frac{\partial P^{ \pm}}{\partial t}+\left(U \mp V_{A r}\right) \frac{\partial P^{ \pm}}{\partial R}+ & \\
\left(\frac{U \pm V_{A r}}{R}\right) P^{ \pm}+M_{\beta \alpha}^{ \pm} F_{\alpha \beta} & =0 \\
\frac{\partial F}{\partial t}+U \frac{\partial F}{\partial R}+\frac{U}{R} F-\left(2 \mathbf{k} \cdot \mathbf{v}_{A}\right) J+ & =0 \\
2\left[M_{\beta \alpha}^{+} P_{\alpha \beta}^{-}+M_{\beta \alpha}^{-} P_{\alpha \beta}^{+}\right] & =0 \\
\frac{\partial J}{\partial t}+U \frac{\partial J}{\partial R}+\frac{U}{R} J+\left(2 \mathbf{k} \cdot \mathbf{v}_{A}\right) F & =0
\end{aligned}
$$

where $P^{ \pm}(\mathbf{R}, \mathbf{k})$ are the energy spectra for the Elsässer fields, $F$ is the energy difference spectrum, and $J$ is referred to as the helicity of the induced electric field. For convenience these definitions, along with a few others, are collected together in Table 1 . The averaging operator $\langle\cdots\rangle$ may be interpreted as an ensemble average or an average over fast fluctuations [Zhou and Matthaeus, 1990a]. As usual, repeated indices imply summation. Also present in the equations are the mixing tensors, $M_{\beta \alpha}^{ \pm}(\mathbf{R})$, which mediate the couplings between the spectra. Their role and nature, discussed in the next section, is central to this work. These linear equations can also be obtained using the coordinate transformation approach [Tu and Marsch, 1990]. Even though essentially the same linear model can be derived by both the multiple scales and coordinate transformation methods, difference exist in how the models treat the fast variations associated with certain rotational symmetries of the fluctuations. The transformation approach [Marsch and Tu, 1993] requires separate derivations to specialize the model to geometries in which these variations either occur or do not occur. In the present multiple scales model, turbulence of var- 
Table 1. Some Useful Definitions Involving the (Scalar) Small-Scale Fields in $k$ Space

\begin{tabular}{ccl}
\hline Symbol & Definition & Description \\
\hline $\mathbf{z}^{ \pm}(\mathbf{k})$ & $\mathbf{v} \pm \mathbf{b} / \sqrt{4 \pi \rho}$ & Elsässer variables \\
$P^{ \pm}(\mathbf{k})$ & $\left\langle\left(\mathbf{z}^{ \pm}\right)^{*} \cdot \mathbf{z}^{ \pm}\right\rangle$ & Elsässer energies \\
& $=2 E \pm 4 H_{c}$ & \\
$F(\mathbf{k})$ & $2 \operatorname{Re}\left\langle\left(\mathbf{z}^{+}\right)^{*} \cdot \mathbf{z}^{-}\right\rangle$ & energy difference \\
& $=4\left(E^{v}-E^{b}\right)$ & \\
$J(\mathbf{k})$ & $2 \operatorname{Im}\left\langle\left(\mathbf{z}^{+}\right)^{*} \cdot \mathbf{z}^{-}\right\rangle$ & electric helicity \\
$\sigma_{c}$ & $\frac{P^{-}-P^{+}}{P^{-}+P^{+}}$ & norm. cross helicity \\
& $=2 H_{c} / E$ & \\
$\sigma_{D}$ & $\frac{F}{P^{-}+P^{+}}$ & norm. energy diff. \\
& $=\frac{E^{v}-E^{b}}{E^{v}+E^{b}}$ & \\
$r_{A}$ & $\frac{E^{v}}{E^{b}}$ & Alfvén ratio \\
& $=\frac{1+\sigma_{D}}{1-\sigma_{D}}$ & \\
$E^{v}(\mathbf{k})$ & $\left\langle\mathbf{v}^{*} \cdot \mathbf{v}\right\rangle / 2$ & kinetic energy \\
$E^{b}(\mathbf{k})$ & $\left\langle\mathbf{b}^{*} \cdot \mathbf{b}\right\rangle / 8 \pi \rho$ & magnetic energy \\
$H_{c}(\mathbf{k})$ & $\operatorname{Re}\left\{\left\langle\mathbf{v}^{*} \cdot \mathbf{b} / \sqrt{4 \pi \rho}\right\rangle\right\} / 2$ & cross helicity \\
\hline
\end{tabular}

All quantities are functions of $\mathbf{R}$ and the small-scale wave vector $\mathbf{k}$, except $\rho=\rho(R)$. An asterisk denotes complex conjugation.

ious symmetries is directly incorporated into a single model. These properties are discussed further below and in later sections.

The mean velocity field is assumed to be radially directed with constant speed $U$, the mean magnetic field $\mathbf{B}_{0}$ is the standard Parker spiral, and the mass density $\rho$ varies as $1 / R^{2}, R$ being the heliocentric distance (see Appendix A for precise forms). The large-scale Alfvén velocity is therefore $\mathbf{V}_{A}=\mathbf{B}_{0} /(4 \pi \rho)^{1 / 2}$. Our convention is to choose $B_{0 r}>0$, so that the $\mathbf{z}^{+}$fluctuations are of inward type and the $\mathbf{z}^{-}$ones of outward type. We caution the reader that the opposite convention is also in use. Hereafter, we refer to this particular choice of fields as the model large-scale fields. These forms preclude the existence of shocks and stream structure in the model solar wind, features which undoubtably influence the evolution of the actual fluctuations. Nonetheless, with suitable choice of $U$ conditions appropriate to the interior of either fast or slow speed streams may be investigated.

Physically, the terms in equations (1)-(3) encompass the effects of advection and expansion of the solar wind, propagating small-scale waves, and mixing. Furthermore, all the terms are linear. In contrast to WKB transport theory, all of these effects, including mixing, are present at leading order. As discussed above, here we focus upon the properties of the linear transport operators, the nonlinear terms having been dropped at the final stage of the derivation. These will be considered in future publications, but recall that in steady fully developed homogeneous turbulence there is no net spectral transport of $P^{ \pm}$in the inertial range $\left(N L^{ \pm}=0\right)$ [cf. Zhou and Matthaeus, 1990c; Matthaeus et al., 1994a].

There are five further points to consider. First, no dissipative terms appear since we are considering inertial range fluctuations and the (direct) effects of viscosity and resistivity are believed to be important only at much smaller scales. Second, we have neglected terms involving index antisymmetric quantities, such as the magnetic helicity [Zhou and Matthaeus, 1990a].

Third, at this point the spectra in (1)-(3) are considered to be modal (dependent upon the full wave vector). In practice, we will present results in terms of either omnidirectional or reduced spectra [e.g., Batchelor, 1970]. The relationships among these types of spectra for various turbulence symmetries are discussed in Appendices A and B. Since most observational data in the solar wind have been obtained using single spacecraft measurements, only reduced spectra are available for comparison with theoretical results. To facilitate such comparisons our earlier work was usually presented in terms of reduced spectra [Zhou and Matthaeus, 1990a; Oughton and Matthaeus, 1992]. In the numerical integrations presented here, however, we employ the omnidirectional forms in order to avoid difficulties connected with imposition of the boundary conditions (see Oughton [1993] for further discussion). Under the assumption of a power law inertial range it is straightforward to calculate the equivalent reduced spectra from the omnidirectional forms; the details are given in Appendix B.

Fourth, to close the model it is necessary to make definite assumptions regarding the rotational symmetry properties of the small-scale turbulence. In this paper the turbulence is approximated as being either isotropic, 2D, or slab; these terms are defined in the results section.

Finally, we comment on the distinction between our multiple scales approach and the decomposition method used by the Lindau group [Marsch and Tu, 1990, 1993; Tu and Marsch, 1990, 1992, 1993]. In their case, two neighboring points in the heliosphere; $\mathbf{X}_{A}$ and $\mathbf{X}_{B}$, are chosen, which represent, in effect, the positions of the experimentalist's probes. A linear transformation of these coordinates is then performed, producing the new coordinates $\mathbf{r}^{\prime}=\mathbf{X}_{B}-\mathbf{X}_{A}, \mathbf{X}^{\prime}=\left(\mathbf{X}_{A}+\mathbf{X}_{B}\right) / 2$. Although $\mathbf{r}^{\prime}$ is always assumed to be small, no explicit scale separation exists in their model, but the probe separation appears explicitly as one of the coordinates. By contrast, in our work we identify the formal scaleseparation parameter with the ratio of two physical length scales: $\epsilon=\lambda_{c} / R$, where $\lambda_{c}$ is the correlation length for the fluctuations and $R$ is the heliocentric distance. The former is (roughly) the spatial scale on which the fluctuations vary, the latter that on which the mean fields change significantly. Observationally, $\epsilon$ is typically much less than unity, particularly beyond about $0.5 \mathrm{AU}$ [e.g., Hundhausen, 1972; Matthaeus and Goldstein, 1982a; Tu et al., 1989]. When we average to 
produce transport equations, all absolute fast coordinate variations disappear. However, in our model, the probe separation (and therefore its Fourier conjugate, the wave vector $\mathbf{k}$ ) is treated from the start as a parameter, not a coordinate. Therefore use of the averaging operator during the formation of transport equations does not lead to the vanishing of dependence on $\mathbf{r}$ (or k). After expansion in $\epsilon$ and equating like powers, we arrive at transport equations in which $\epsilon$ does not appear. However, independent parameters that depend upon $\mathbf{k}$ remain in the transport equations. These may be large or small in accordance with the symmetry of the fluctuations, which in our approach is controlled independently of the magnitude of the scale separation parameter $\epsilon$. We return to this issue in later sections.

In closing this section, we stress that no approximations regarding the relative abundances of the inwardand outward-type fluctuations have been made: the model supports completely arbitrary admixtures of these modes. Additionally, while we have chosen particularly simple forms for the model large-scale fields, the model itself does not require the approximations to be so basic. In the future, we envisage adopting more realistic approximations to the solar wind's observed large-scale fields.

\section{Mixing and the Transport Equations}

If the $M_{\beta \alpha}^{ \pm}$in (1) were absent, the \pm energy spectra would decouple and evolve independently in accord with WKB theory, while (2) and (3) would be largely irrelevant. When $M_{\beta \alpha}^{ \pm} \neq 0$, i.e., there are gradients in the large-scale fields, we expect coupling between the \pm fields (here called mixing), mediated by the energy difference spectrum $F_{\alpha \beta}$, unless something acts to short circuit these couplings. To a large extent this paper is concerned with illustrating how important mixing can be in the context of the linear transport of solar wind fluctuations.

A few words are in order regarding the nature of the mixing tensors. Mathematically, they mediate couplings between the independent small-scale fields; physically, they may be interpreted as acting to scatter the $\mathbf{z}^{ \pm}$modes off large-scale gradients of the mean fields. Another interpretation is associated with the MHD ana$\log$ of the Reynolds stresses [e.g., Tennekes and Lumley, 1972]. In this view the mixing tensors are a sort of mean rate of strain in the large-scale fields, from which energy may be fed to smaller scales. Note that the tensors vanish when the large-scale fields are uniform.

The structure of (1)-(3) heuristically reveals how mixing might be either important or a very small effect. For nonuniform large-scale fields, mixing requires nonvanishing $F_{\alpha \beta}$. However, the rate of change of $F_{\alpha \beta}$ includes terms like $\mathbf{k} \cdot \mathbf{V}_{A}$ (cf. equation (2)) that, along with analogous terms in (3) can act to produce rapid oscillations, and therefore values of $F_{\alpha \beta}$ that average quickly to zero. On the other hand, when $\mathbf{k} \cdot \mathbf{V}_{A}$ is very small, or if $J$ is maintained at zero, this oscillatory effect becomes weak and cannot prevent mixing.
As it happens, imposition of symmetries such as twodimensionality (wave vectors perpendicular to $\mathbf{V}_{A}$ ) or isotropy have precisely these effects. For slab symmetry the oscillatory terms are strong and act to enforce WKB theory [Matthaeus et al., 1994b; Marsch and Tu, 1993; Tu and Marsch, 1993].

It also clear that the index symmetry of the mixing tensors enters this discussion. The mixing term in (7) involves a fully contracted product of two tensors. Thus the antisymmetric part of the energy difference tensor couples to the trace of the Elsässer energy spectra when the mixing tensors have an antisymmetric part. It would follow that equations would be needed for all the independent components of the fluctuation spectral tensors. Here we choose to ignore all antisymmetric parts of the spectral tensors, including magnetic and kinetic helicities and the induced electric field. This avoids considerable complication.

In their full generality, the mixing tensors have the form

$$
\begin{aligned}
M_{j i}^{ \pm}(\mathbf{R})=\frac{\partial \mathbf{U}_{i}}{\partial \mathbf{R}_{j}} & \pm \frac{1}{\sqrt{4 \pi \rho}} \frac{\partial \mathbf{B}_{0 i}}{\partial \mathbf{R}_{j}} \\
& -\frac{1}{2} \delta_{i j} \nabla \cdot\left(\frac{\mathbf{U}}{2} \pm \mathbf{V}_{A}\right)
\end{aligned}
$$

which is completely determined by the large-scale gradients of the mean fields. However, because these tensors always appear coupled to small-scale spectral tensors (e.g., $Q_{j \alpha} M_{\alpha i}^{ \pm}$), mixing also depends on the nature and rotational symmetry properties of the small-scale turbulence. Assuming that this small-scale turbulence is either isotropic, 2D, or slab enables the trace of $Q_{j \alpha} M_{\alpha i}^{ \pm}$ to be evaluated and written as the product of two scalar functions: $M^{ \pm} Q$, where $Q=Q_{\alpha \alpha}$, and the $M^{ \pm}$are effective mixing operators. The choice of spectrum type also plays a role in determining the forms of the $M^{ \pm}$. In Appendix $A$ it is shown that the effective mixing operators appropriate for use with omnidirectional spectra and the large-scale fields described above are

$$
\begin{aligned}
M_{\text {iso }}^{ \pm}= & \frac{1}{2 R}\left[\frac{U}{3} \mp V_{A r}\right] \\
M_{2 D}^{ \pm}= & \frac{1}{2 R}\left[U \cos ^{2} \psi \pm V_{A r}\left(3 \cos ^{2} \psi-2\right) \pm\right. \\
& \left.\frac{V_{A r 0}}{2}\left(\frac{\Omega R_{0}}{U}\right)\left(2-\frac{3 R_{0}}{R}\right) \sin \theta \sin 2 \psi\right] \\
M_{\text {slab }}^{ \pm}= & M_{2 D}^{ \pm}
\end{aligned}
$$

where $\psi$ is the winding angle of the Parker spiral magnetic field [Parker, 1958], $\Omega$ the solar rotation rate, $V_{A r}=V_{A r 0} R_{0} / R$ the radial component of $\mathrm{V}_{A}$, and $R_{0}$ a reference radius taken equal to $10 R_{\text {Sun }}$. The equality of the $2 \mathrm{D}$ and slab mixing operators is a consequence of the formal (structural) equivalence of the omnidirectional spectra for the two cases. In both situations, the fluctuation energy is all in spectral tensor components perpendicular to $\mathbf{B}_{0}$, but the interpretations are distinctly different (e.g., Appendix C of Oughton [1993]). 
As suggested by Figure 1 , when $R \gtrsim 2$ AU the effective operators are all essentially the same, being asymptotically $\sim 1 / R$. Inside $1 \mathrm{AU}$, however, important qualitative differences exist between both the plus and minus versions for the same type of turbulence, and also between $M^{ \pm}$for different types of rotational symmetry. In particular, when $V_{A r 0}>U / 3$, the isotropic forms are qualitatively different from the others, with $M_{\text {iso }}^{+}$ actually starting negative, crossing over to a positive peak at a few $R_{0}$, and thereafter decreasing towards zero. For $2 \mathrm{D}$ and slab turbulence, the $\mathrm{M}^{+}$operators are strictly monotonically decreasing from positive initial values. As will be demonstrated in the results section, the anomalous behavior of $M_{\mathrm{iso}}^{+}$is largely responsible for the strongly reduced mixing of isotropic turbulence relative to $2 \mathrm{D}$ turbulence.

In the solar wind, the gradients in the mean fields are largest in the inner heliosphere, so that the components of the mixing tensors are also largest in magnitude in this region. Furthermore, since the mixing operators act as nonconstant coupling coefficients between the smallscale fields, it seems likely that any significant influence exerted by the mixing tensors on the radial evolution of the fluctuating quantities will also occur close to the sun. In support of this statement we have compared the magnitudes of the various coefficients of (1)-(3) for 2D turbulence (the case for which the mixing operators are largest). Within the first few tenths of an $\mathrm{AU}, M_{2 D}^{+}$ differs from the other coefficients by no more than a factor of two (either way). By $1 \mathrm{AU}$, however, it is only a quarter of the other coefficients and at 2 AU barely a tenth.

Zhou and Matthaeus [1990b] and Matthaeus et al. [1994b] have identified three situations (not necessarily mutually exclusive) in which mixing effects can be important: (1) $\mathbf{k} \cdot \mathbf{V}_{A} \approx 0,(2) V_{A} / U \approx 0$, and (3) the fluctuations do not obey a (linear) wave dispersion relation, e.g., broadband turbulence. Clearly, these cases satisfy the heuristic conditions for mixing discussed above. The crucial feature regarding the first two cases is that even when the fluctuations have wavelengths which are arbitrarily small (the usual condition for the applicability of WKB theory), there are situations in which the fluctuation frequencies are also small. The expansion

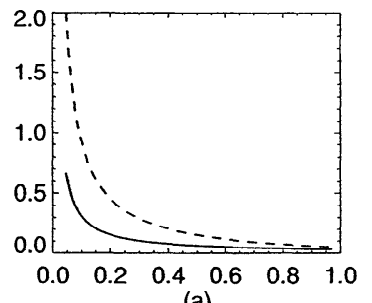

(a)

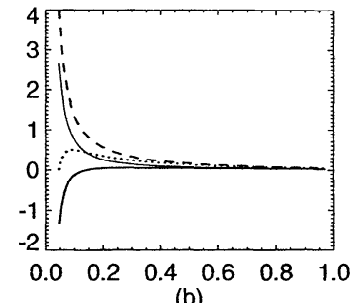

(b)
Figure 1. Plots of the effective mixing operators as a function of heliocentric distance in AU. (a) The case $V_{A r 0}=0$ (for which $M^{+}=M^{-}$), (b) the case $V_{A r 0}=U$. The isotropic versions are plotted using solid lines, and the 2D forms using dashed lines. In (b) the $M^{-}$forms are indicated by the thinner solid line and the dotted line. The inner boundary is located at $R_{0}=10 R_{\text {sun }}$. parameter based on the frequency is then not small (cf. $\epsilon_{1}^{\prime}$ in the work by Zhou and Matthaeus [1990b]), and it is inappropriate to use the standard leading order WKB transport equation(s).

We are particularly interested in how the radial evolution of the physical quantities is affected by the size of $\mathbf{k} \cdot \mathbf{V}_{\boldsymbol{A}}$. This factor is essentially the coupling strength between the $F$ and $J$ fields and may be thought of as a "WKB-enforcing" term. If $\mathbf{k} \cdot \mathbf{V}_{A} \approx 0$, then $F$ and $J$ are only weakly coupled and strong mixing occurs. The initial dominance of the outward-type modes causes growth of $F$, which in turn causes growth of the inward-type modes, leading to significant decreases in $\sigma_{c}=\left(\boldsymbol{P}^{-}-\boldsymbol{P}^{+}\right) /\left(\boldsymbol{P}^{-}+\boldsymbol{P}^{+}\right)$with heliocentric distance. However, when $\mathbf{k} \cdot \mathbf{V}_{A} \not \approx 0, F$ and $J$ are strongly coupled. For such cases $F$ is constrained to oscillate about zero with small amplitude and thus is ineffective in coupling $P^{+}$to $P^{-}$. The energy in the inward-type fluctuations consequently remains a tiny fraction of that in the outward-type and WKB-like solutions are obtained.

\section{Results}

In order to solve the equations we have, in most cases, resorted to numerical techniques, namely Chebyshev spectral (collocation) methods [Gottlieb and Orszag, 1977; Canuto et al., 1988], the equations being integrated in time to steady-state solutions. Boundary conditions are imposed at $R_{0}=10 R_{\text {Sun }}$ and chosen so that the fluctuations there are purely outwardly propagating, i.e., $P_{0}^{-}>0, P_{0}^{+}=F_{0}=J_{0}=0$, where the zero subscript indicates evaluation at $R_{0}$. Furthermore, because the equations being solved are linear, the solutions can be multiplied by an arbitrary constant. For convenience we always choose $P_{0}^{-}=1$. These will be referred to as "standard" boundary conditions, and their use allows (but does not require) the inner boundary to be interpreted as the Alfvén critical radius $\left(R_{c}\right)$. This is the distance at which the (radial) flow velocity becomes equal to the (radial) Alfvén velocity, with theory and observations suggesting that it lies in the range 7$30 R_{\text {Sun }}$ [Weber and Davis, 1967; Pizzo et al., 1983; Marsch and Richter, 1984; Lotova et al., 1985; Lotova, 1988]. The conventional perspective regarding the critical point is that it prevents inward-type fluctuations from escaping past it, their net velocity being towards the sun. Thus only outward-type fluctuations should exist at $R_{c}$. This view has been challenged by Hollweg [1990], who, examining the higher order corrections to a regularized WKB expansion, concludes that a small admixture of inward waves is required at the critical point. However, a more general scale separated approach [Matthaeus et al., 1994b] reveals that the WKBtype solutions are special cases, which lack mixing effects at leading order and to which Hollweg's comments apply. For more general solutions that permit mixing, it is consistent to choose boundary data with purely outgoing waves at the critical point. Here we usually adopt the standard boundary conditions, even when $V_{A \tau 0}<U$. 
A major objective of the following subsections will be to indicate some of the circumstances in which mixing effects are important. As a shorthand we will sometimes refer to the mixing effects as "strong," meaning that the solutions obtained under the specified conditions are significantly different from the equivalent leading order WKB ones. Similarly, "weak" will indicate that mixing effects are relatively unimportant, and thus that the solutions are close to the WKB forms.

Steady state solutions to (1)-(3), with the nonlinear terms dropped, depend on the large-scale speeds only through the three ratios (1) $A_{0}=V_{A r 0} / U$, (2) $\left|\hat{\mathbf{k}} \cdot \mathbf{V}_{A}\right| / U$, and (3) $\Omega R_{0} / U$, the last determining the degree of winding of the magnetic field. For isotropic turbulence, only the first ratio comes into play, so that the solutions are independent of the absolute values of $U$ and $\mathbf{v}_{A}$. Since the equations are linear we will only present solutions for one value of $U$, equivalent to $400 \mathrm{~km} / \mathrm{sec}$, which is about the average solar wind speed. Values of $V_{A r 0} / U$ used lie between 0 and 1 inclusive. When $V_{A r 0} / U=0$ there is no large-scale magnetic field present, so that conditions are similar to those which would exist in a planar current sheet. At the other extreme, when $V_{A r 0} / U=1$, the inner boundary corresponds to an Alfvénic critical radius. Intcrmediate values may be interpreted as the inner boundary lying beyond this critical radius.

In the limit that $V_{A} / U$ approaches zero, analytic solutions for the steady linear transport model may be obtained. For the isotropic case such solutions were first obtained by Zhou and Matthaeus [1990a]; an identical approach furnishes analytic solutions for the 2D and slab cases, which the codes faithfully reproduce [Oughton, 1993]. We wish to emphasize that these analytic solutions, though oversimplified and perhaps in some cases unphysical, have served to illustrate the types of behavior that can be introduced by the mixing terms in the transport equations, while also providing control solutions for the numerics. We discuss the interpretation of these solutions in the final section.

Note that near the critical point $\left(V_{A r}=U\right)$ the behavior of the coefficients for the advection and expansion terms is quite different for the inward- and outward-type fields (equation (1)). More specifically, in this region the outward-type modes undergo strong advection but relatively weak expansion, while the situation is reversed for inward-type fluctuations. Speaking very loosely, the growing bubbles of inward-type modes are left behind by jets of outward-type fluctuations.

We will present the numerical results using plots of spectral amplitudes for $P^{ \pm}, \sigma_{c}$, and $\sigma_{D}$, all as functions of heliocentric distance. The normalized cross helicity $\sigma_{c}$ is interpretable as a measure of the relative abundances of inward- and outward-type modes, the extremal values $\sigma_{c}= \pm 1$ corresponding to purely outward- and purely inward-type modes, respectively. It is also a normalized correlation coefficient for $\langle\mathbf{v} \cdot \mathbf{b}\rangle$, so that it conveys information on the degree of alignment existing between the small-scale fields. The normalized energy difference $\sigma_{D}$ is, as its name suggests, the ratio of the difference in kinetic and magnetic energy to their sum. The same information is contained in the Alfvén ratio, $r_{A}=E^{v} / E^{b}$; however, the bounded character of $\sigma_{D}$ makes it a more convenient quantity to plot. For isotropic and 2D turbulence, the spectral amplitude is evaluated at a fixed but arbitrary inertial range wavenumber. This approach is possible because there is no explicit $k$ dependence in the linear equations for turbulence of these types. In contrast, the slab geometry is $k$ dependent at the linear level, and so the particular inertial range wavenumber used is important. Also, the linear transport equations do not contain nonlocal or derivative expressions involving $\mathbf{k}$, so no wavenumber boundary conditions are required. (Generally speaking, when nonlinear effects are included, spectral transfer communicates excitations from the edge of the inertial range, where boundary conditions are needed, throughout $k$ space. Differing wavenumbers then cease to be independent.)

Since at this level of approximation the transport equations are linear, the various solutions may be superposed. Thus we can obtain the evolution of various admixtures of fluctuations, for example, a 2D-slab mixture. Recent research regarding the nature of solar wind fluctuations [Matthaeus et al., 1990; Bieber et al., 1994], from various perspectives, suggests that such composite models are a better approximation to the true system when compared to fluctuations characterized by a single rotational symmetry property. We now examine results for the three basic types of fluctuations and then consider a few linear superpositions of these solutions and how they compare with observational data.

\section{Isotropic Fluctuations}

The steady state equations for fluctuations which are isotropically distributed, with the above choice of model large-scale fields, are

$$
\begin{aligned}
\left(U \mp V_{A r}\right) \frac{\partial P^{ \pm}}{\partial R}+\left(\frac{U \pm V_{A r}}{R}\right) P^{ \pm} & \\
+M_{\mathrm{iso}}^{ \pm} F & =0 \\
U \frac{\partial F}{\partial R}+\frac{U}{R} F+2\left[M_{\mathrm{iso}}^{+} P^{-}+M_{\mathrm{iso}}^{-} P^{+}\right] & =0
\end{aligned}
$$

where $M_{\text {iso }}^{ \pm}$is given by (5). Because homogeneity requires that $J(\mathbf{k})$ be an odd function of $\mathbf{k}$, the function $J(\mathbf{k})$ must vanish identically for turbulence which is isotropic and nonhelical [Oughton and Matthaeus, 1992; Oughton, 1993]. We stress that no directional averaging is performed to arrive at this conclusion $-J$ vanishes as a consequence of the assumption of isotropy. Consequently, the WKB enforcing term cannot come into play and mixing is strong. As can be seen below, this leads to substantial falloff in the normalized cross helicity with increasing heliocentric distance. The solutions also show significant dependence on the value of $A_{0}$, with mixing becoming weaker as $A_{0}$ increases from 0 to 1.

It should be noted that, by itself, linear transport cannot maintain isotropy of the fluctuations, since the 
structure of the equations breaks the isotropic symmetry. Some agency which continually isotropizes the fluctuations (e.g., strong turbulence) must therefore be active in order to maintain consistency with the underlying assumptions. This is a feature common to all cases in which a symmetry of the fluctuations is assumed. However, in the isotropic case, the assumption rules out an approach to WKB behavior. For slab fluctuations nonzero $J$ is permitted, leading to very different behavior.

Figure 2 shows omnidirectional spectral amplitudes for two isotropic cases, $V_{A r 0} / U=0$ and 1 , with standard boundary conditions in place. Clearly, when $V_{A r 0} / U=1$ (or more generally when it is big enough to cause a sign change in $\mathrm{M}^{+}$), there is a strong tendency for the turbulence to contain a sizable excess of small-scale kinetic energy just above the critical point $\left(\sigma_{D} \approx 0.2\right)$. However, within a few critical radii the situation reverses and magnetic energy is favored at inertial range scales $\left(\sigma_{D} \approx-0.3\right)$. This special character of the transport just above the critical point may be associated with a possible local enhancement of velocity field turbulence inferred from observations [e.g., Lotova et al., 1985; Lotova, 1988; Mullan, 1990] within a few critical radii.

When the turbulence is isotropic, the reduced and omnidirectional spectra differ only by a multiplicative constant (assuming a power law form for the latter, see Appendix B). Because of this similarity we do not show plots of the reduced spectra for the isotropic case.

Nonstandard boundary conditions have also been investigated. The equations are relatively insensitive to the value chosen for the boundary condition on inwardtype fluctuations, provided the energy difference at $R_{0}$ is still held at zero. For such conditions, choosing $P_{0}^{+} / P_{0}^{-} \gtrsim 0.005$ leads to the immediate plummeting of
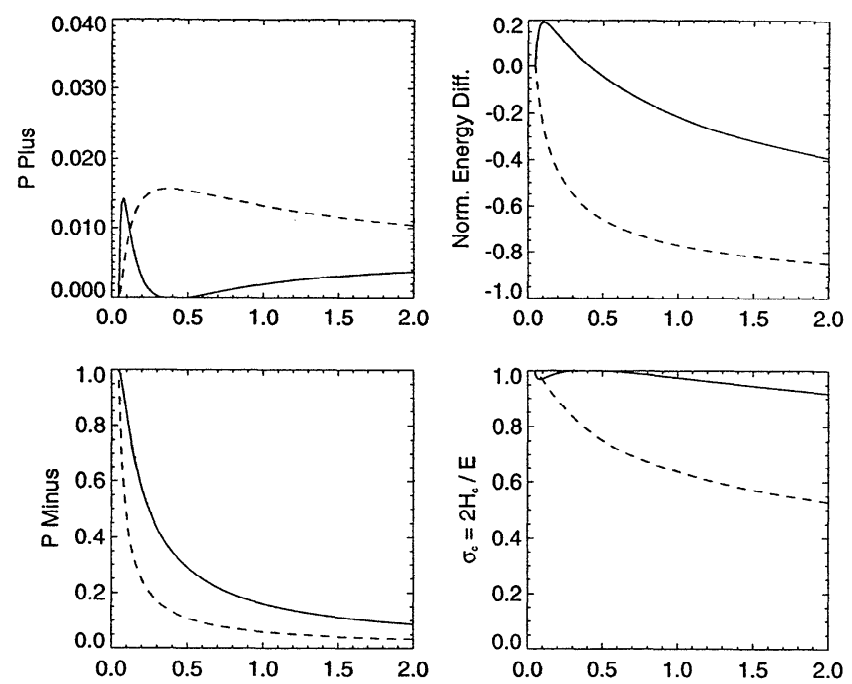

Figure 2. Radial evolution of various omnidirectional spectral densities for isotropic turbulence (at fixed inertial range wavenumber). Standard boundary conditions are imposed. The horizontal coordinate is heliocentric distance (AU). Solid curves: $V_{A r 0} / U=1$. Dashed curves: $V_{A r 0} / U=0$.
$P^{+}(R)$ to a value which is approximately the same one it has when $P_{0}^{+}$is zero, In other words, enforced equipartition of the small-scale energy at $R_{c}$ is associated with at most a tiny fraction $\left(\sim 10^{-3}\right)$ of inwardtype modes relative to outward-type ones just above the critical point. The subsequent evolution of $P^{+}$is practically indistinguishable from that occurring when $P_{0}^{+}=0$.

When the equipartition restriction at $R_{c}$ is relaxed, however, visible changes in the radial evolution of the fields ensue. The choice of $F$ is not completely arbitrary for such cases as the energy difference is constrained by several inequalities. First, using the definition of $F$ and the Schwartz inequality it can be shown that $|F| \leq 2\left(P^{+} P^{-}\right)^{1 / 2}$. Second, since the energy difference cannot be greater than the total energy we must also have $|F| \leq P^{+}+P^{-}$. Figure 3 shows the situation for $P_{0}^{+}=0.01, P_{0}^{-}=1$, and two choices of $F_{0}$, namely 0.1 and 0.2 , the latter number being $F_{0}$ 's maximum allowed value for these conditions. While negative values of $F_{0}$ are not mathematically ruled out, we have elected not to investigate these cases since the equations tend to drive $F$ positive in this region. It can be seen that for fixed $P_{0}^{ \pm}$, increasing the kinetic energy excess at the boundary leads to mildly weaker mixing at a given heliocentric distance, with no significant change in the evolutionary trends.

\section{Two-Dimensional Fluctuations}

We define 2D turbulence by the following properties: (1) $\mathbf{k} \perp \mathbf{B}_{0}$, (2) the fluctuations are perpendicular to both $\mathbf{k}$ and $\mathbf{B}_{0}$, and (3) the fluctuations are distributed isotropically in the planes normal to $\mathbf{B}_{0}$. As a consequence of this geometry $\mathbf{k} \cdot \mathbf{V}_{A} \equiv 0$, and $F$ and $J$ are again decoupled, as in the isotropic case. Accordingly, the WKB-enforcing terms are absent and mixing is always strong. In fact, $J$ is again identically zero by symmetry, for reasons which are essentially the same as those applying in the 3D isotropic case. Thus, except
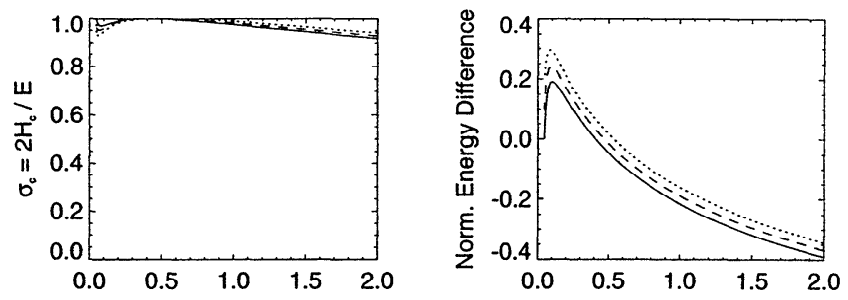

Figure 3. Radial evolution of the normalized cross helicity and normalized energy difference of isotropic turbulence for some nonstandard boundary conditions (omnidirectional spectral amplitude at fixed wavenumber). In each case, $V_{A r 0}=U$. The evolution for two initial values of $F$ is shown when $P^{+}=0.01, P^{-}=1: F$ $=0.1$ (dashed curve), $F=0.2$ (dotted curve). The solid curves are the solutions for standard boundary conditions. Solutions for $P_{0}^{+}=0.01, P_{0}^{-}=1$, and $F_{0}=0$, are visually indistinguishable from the standard case when plotted at this scale. Once again, the horizontal coordinate is heliocentric distance $(\mathrm{AU})$. 
for the mixing operators, given now by (6), the steady state transport equations for $2 \mathrm{D}$ fluctuations are the same as those for the isotropic case, (8) and (9), so we do not reproduce them here. Before presenting the results we note two points regarding the relevance of $2 \mathrm{D}$ fluctuations in the solar wind.

Results from simulation studies of incompressible 2D and 3D MHD turbulence with a mean magnetic field $\left(\mathbf{B}_{0}\right)$ present, have shown that in such geometries, energy is transferred to the perpendicular wave vector components of the fluctuations much more rapidly than it is to the parallel ones [Shebalin et al., 1983; Hossain et al., 1985; Oughton et al., 1994a, b]. In other words the 2D spectrum "switches on" first. This suggests that 2D fluctuations may be relevant to the solar wind system with its approximately Parker spiral $\mathbf{B}_{0}$. Indeed, there is also some observational evidence that the solar wind can be modeled as an admixture of slab $\left(\mathbf{k} \| \mathbf{B}_{0}\right)$ and 2D fluctuations [Matthaeus et al., 1990], a possibility which has been discussed in the context of transport theory by Oughton and Matthaeus [1992], Tu and Marsch [1992, 1993], and Marsch and Tu [1993]. We consider this multiple component model further below.

Furthermore, the theoretical analysis of nearly incompressible MHD turbulence [Zank and Matthaeus, 1992a, $\mathrm{b}, 1993$ ], also suggests that when the plasma beta is of order unity (as it is in much of the solar wind), the turbulence may consist of two components: (1) Alfvén waves propagating along $\mathbf{B}_{0}$, and (2) incompressible $2 \mathrm{D}$ MHD turbulence in the surfaces perpendicular to $\mathbf{B}_{0}$. In the solar wind such 2D surfaces would be advected outward by the supersonic flow (see also Tu and Marsch [1992, 1993], Marsch and Tu [1993]). At higher orders, nonlinear interactions between the two components can also occur [Zank and Matthaeus, 1992b, 1993].

Assuming that the omnidirectional spectrum $Q^{\text {omni }}$ is a power law, with spectral slope $-\alpha$, it is possible to calculate the equivalent reduced spectrum $Q^{\text {red }}$. The details are given in Appendix B, with the result that for 2D turbulence

$$
\begin{aligned}
Q^{\mathrm{red}}\left(k_{1}\right) & =\frac{I(\alpha)}{\pi \sin \psi} Q^{\mathrm{omni}}\left(\frac{k_{1}}{\sin \psi}\right) \\
& =\frac{I(\alpha) \sin ^{\alpha-1} \psi}{\pi} Q^{\mathrm{omni}}\left(k_{1}\right)
\end{aligned}
$$

where $k_{1}$ is equivalent to the radial component of $\mathbf{k}$, and $I(\alpha)$ is a pure number. In the first line, the argument of the omnidirectional spectrum, $k_{1} / \sin \psi$, may be interpreted as the component of $\mathbf{k}$ lying in the plane of $\mathbf{B}_{0}$ and $\mathbf{R}$. Thus, the reduced spectra of $2 \mathrm{D}$ turbulence contain a purely geometrical factor which depends on the local winding angle. In general, these geometrical correction factors alter not just the level, but also the radial profile of the raw spectral densities. However, normalized quantities (e.g., $\sigma_{c}, r_{A}$ ) may be computed from either $Q^{\text {red }}$ or $Q^{\text {omni }}$, and will be independent of such common factors. Tu and Marsch [1992, 1993] have discussed the closely related issue of the influence on observed reduced spectra of the angle between the sam- pling direction and $\mathbf{B}_{0}$, for models wherein the fluctuations are divided into slab Alfvén waves and so-called convective structures.

We turn now to numerical solutions of the transport model for 2D fluctuations. To facilitate interpretation of our subsequent results, we compare the reduced (i.e., observed) spectral amplitudes at some fixed inertial range $k_{1}$, with the underlying omnidirectional spectra at the corresponding wavenumber. The lefthand panels of Figure 4 show the two spectral forms of $P^{ \pm}$considered in this section, for the usual situation of standard boundary conditions, with $V_{A r 0} / U=1$. The curves shown are for the Kolmogorov case of $\alpha=\frac{5}{3}$. As $I(\alpha) / \pi$ only varies between $\frac{1}{2}$ for $\alpha=1$ and $1 / \pi$ for $\alpha=2$, the dependence of this factor on the spectral slope is relatively weak, it does however determine the amplitude at large distances, where $\sin \psi \approx 1$. In the region close to $R_{0}, \sin \psi$ is small, so that, relative to the omnidirectional spectra, the reduced spectral densities are greatly diminished. In fact, the reduced spectral amplitudes are lower than the corresponding omnidirectional ones at all distances, but, the difference between the two forms decreases as $R$ increases. When comparing the model solutions to observational data, it will be important to keep points like this in mind.

The right-hand panels of Figure 4 show the radial evolution of the normalized forms of the cross helicity and energy difference, for the two cases $V_{A r 0} / U=0.2$ and 1 (standard bc's). For 2D turbulence, the dependence of the normalized quantities on this parameter is evidently somewhat weaker than for the isotropic case, particularly away from $R_{0}$. The reason is that, unlike
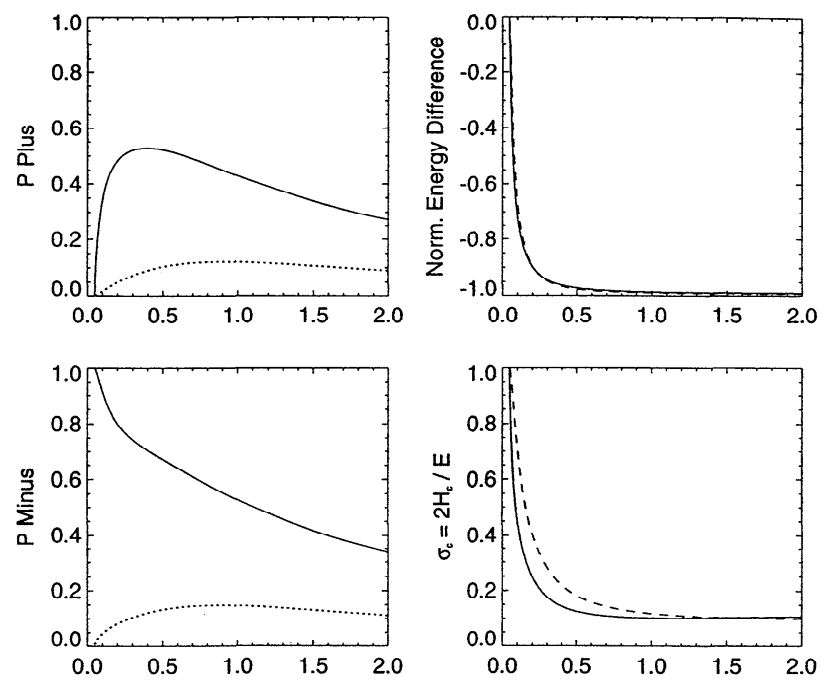

Figure 4. Radial evolution of various spectral densities for 2D turbulence (at an arbitrary inertial range wavenumber). All plots are for standard bc's. The lefthand panels show the omnidirectional (solid curves) and reduced (dotted curves) spectra for the case $V_{A r 0}=U$. The right-hand panels display the normalized cross helicity and normalized energy difference for the two cases $V_{A r 0} / U=0.2$ (dashed curves) and 1 (solid curves). As usual, the horizontal coordinate is heliocentric distance in $\mathrm{AU}$. 
$M_{\text {iso }}^{+}, M_{2 D}^{+}$does not undergo a sign change as $V_{A r 0} / U$ is increased, so that the mixing does not counteract itself. Note that beyond 0.5 AU the small-scale energy is almost entirely magnetic in character. Why this rapid evolution towards magnetic energy dominance occurs is currently not well understood, although some insights may be gained from $V_{A}=0$ analytical models [ $T u$ and Marsch, 1993; Zhou and Matthaeus, 1990a; Oughton, 1993].

For nonstandard boundary conditions on the fields, no qualitative and only small quantitative differences are seen in the radial evolution of any of the spectral densities. For example, when $V_{A r 0}=U, P_{0}^{-}=1$, $P_{0}^{+}=0.01$, and $F_{0}=0,0.1$, or 0.2 , the normalized cross helicity and Alfvén ratio increase slightly as $F_{0}$ is varied from zero up to its maximum allowed value for these conditions $(0.2)$, indicating a minor weakening of mixing. Negative values of $F_{0}$, on the other hand, cause a marginal increase in the strength of mixing, relative to the $F_{0}=0$ case. This is easily understood: the strength of the mixing effect, and in particular growth of $P^{+}$, depends upon the size of $F$. At a given distance, the mixing term in the transport equation for $P^{+}$is more effective for more negative values of $M^{-} F$. As $M^{-}>0$ for all $R>R_{0}$, initially positive values of $F$ cause a brief decay of $P^{+}$, and delay the onset of its growth phase.

In summary, the solutions for two- and three-dimensional isotropic turbulence are quite similar in form, particularly beyond a few tenths of an AU. In both cases approximate saturation of the normalized quantities occurs, typically at a distance of about $1 \mathrm{AU}$. However, because $M_{2 D}^{+}$is strictly positive, the mixing effect is substantially stronger for $2 \mathrm{D}$ turbulence. In fact, the mixing effect for $2 \mathrm{D}$ fluctuations is the strongest that we have seen in any solutions of linear transport equations, regardless of fluctuation symmetry.

\section{Slab Fluctuations}

In the slab geometry the fluctuations are in the plane perpendicular to the wave vector $\mathbf{k}$, with $\mathbf{k}$ parallel to $\mathbf{B}_{0}$. The appropriate steady state equations are

$$
\begin{gathered}
\left(U \mp V_{A r}\right) \frac{\partial P^{ \pm}}{\partial R}+\left(\frac{U \pm V_{A r}}{R}\right) P^{ \pm} \\
+M_{\text {slab }}^{ \pm} F=0 \\
\begin{array}{c}
U \frac{\partial F}{\partial R}+\frac{U}{R} F-\left(2 \mathbf{k} \cdot \mathrm{V}_{A}\right) J \\
+2\left[M_{\text {slab }}^{+} P^{-}+M_{\text {slab }}^{-} P^{+}\right]=0 \\
U \frac{\partial J}{\partial R}+\frac{U}{R} J+\left(2 \mathbf{k} \cdot \mathbf{v}_{A}\right) F=0
\end{array}
\end{gathered}
$$

with $M_{\text {slab }}^{ \pm}$given by (7). Physically, this geometry is associated with Alfvén waves propagating along $\mathbf{B}_{0}$. In contrast to the two previous geometries, $J$ is no longer forced by symmetry to be identically zero, so that the WKB-enforcing term can come into play.
Figure 5 shows an example for the case when $V_{A r 0} / U$ is unity; standard boundary conditions apply and $k_{1}=$ 50 in code units. In physical units this corresponds to a wavenumber of $50 \times 2 \pi / R_{0} \approx 7 \times 10^{-6} \mathrm{~km}^{-1}$, equivalent to a wavelength of about $1 / 1000 \mathrm{AU}$, well within the typical inertial range. The solutions are very close to the leading order WKB results for the same boundary conditions. That is, at all distances $\sigma_{c} \approx 1$ and $r_{A} \approx 1$ $\left(\sigma_{D}=0\right)$.

Evident in Figure 5 are the oscillations described in section 3 as "WKB-enforcing," and their role in attaining that limit is clear here. When $\mathbf{k} \cdot \mathbf{V}_{A}$ is nonzero, $F$ and $J$ exhibit tightly coupled oscillations, $F$ about zero and $J$ about a relatively small positive value which tends to zero as $R \rightarrow \infty$. As $F$ is always small, mixing effects are effectively turned off because at this order $P^{+}$ can grow only weakly if $F \approx 0$ (physically, $F$ is small because Alfvén waves exhibit near equipartition of kinetic and magnetic energy). The energy in the inward-type fluctuations therefore remains a tiny fraction of that in the outward-type ones, leading to recovery of WKB-like solutions. As $R$ increases, the wavelength of the oscillations in $F$ and $J$ also increases because of the dependence of $V_{A}$ on $R$. Spectra for $F$ and $J$, obtained from Helios observations, are shown in Figure 6 of Marsch and $T u$ [1990].

We also emphasize at this point that the presence or absence of the WKB enforcing oscillations is clearly connected with the choice of fluctuation geometry and its effect upon the parameter $\mathbf{k} \cdot \mathbf{V}_{A}$. The structure of the transport equations in their general form was established by expansion in $\epsilon=\lambda_{c} / R$, which is clearly a separate and independent physical quantity. The appearance of rapid time (or space) variations is not connected with the scale separation of the overall problem, rather, its magnitude varies independently in accordance with
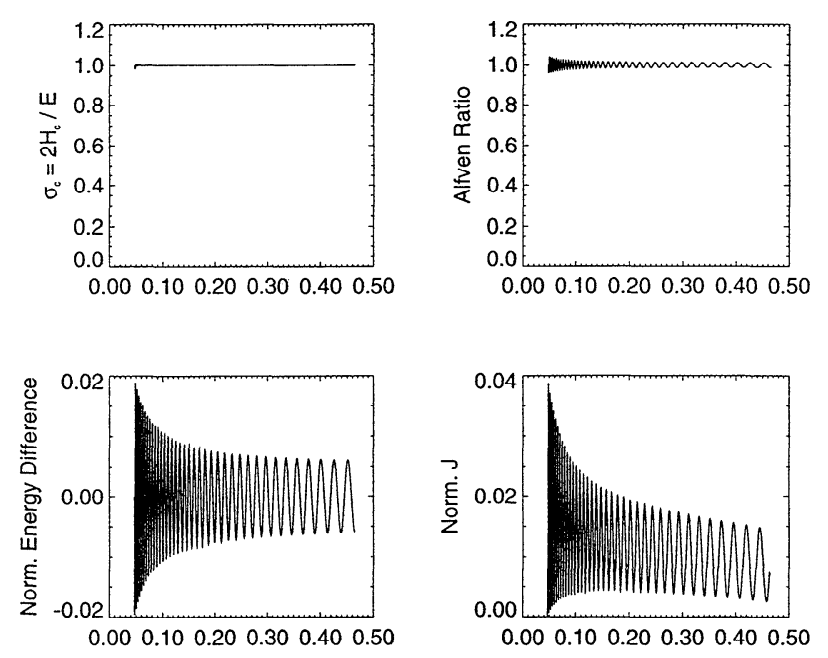

Figure 5. The radial evolution, or lack thereof, of various normalized quantities for slab fluctuations (spectral amplitudes at $\left.k_{1}=50\right)$. The solutions are for standard bc's, and $V_{A r 0}=U$. The lower plots should actually be plotted using vertical bounds of \pm 1 , but such plots deemphasize the oscillations. The horizontal coordinate is heliocentric distance in $\mathrm{AU}$. 
the direction of the excited wave vectors $\mathbf{k}$. Since this effect is of independent physical origin, there is no difficulty inherent in these rapid oscillations. However, even though the formal structure of the transport equations permits these rapid variations, it is clear that practical difficulties arise with regard to considering such solutions to be locally spatially homogeneous.

Figure 6 indicates that the recovery of WKB-like solutions occurs for all values of $k_{1} \gtrsim 2$ (code units); this wavenumber corresponds to a physical wavelength $\sim 1 / 40 \mathrm{AU}$, which is about the same as the observed correlation length for the total energy at $1 \mathrm{AU}$. It follows that since solutions for scales much longer than those characteristic of inertial range fluctuations are WKBlike, the entire inertial range will also behave in this fashion. Clearly, nonzero values of $\mathbf{k} \cdot \mathbf{V}_{A}$ strongly inhibit mixing. This dependence on $\mathbf{k} \cdot \mathbf{V}_{A}$ is essentially what was seen in Heinemann and Olbert's [1980] investigation of the full wave equation (for toroidal waves). For their simplified geometry, where both the large-scale flow and the large-scale magnetic field are radially directed, convergence of the (small amplitude) solutions to WKB forms was reported when $k$ is not unduly small. Convergence to WKB solutions has been discussed recently in more detail by Matthaeus et al. [1994b].

Slab solutions (omnidirectional) for the separate cases of $\mathbf{k}=0$ and $\mathbf{V}_{A}=0$, are highly reminiscent of the 2D forms. When we recall that the two geometries have the same effective mixing operators, this similarity is unsurprising; indeed the same analytic solution, showing strong mixing, holds for both types of fluctuations when $V_{A}=0$ (however, these cases are not of much physical relevance for $2 \mathrm{D}$ and slab fluctuations). If we instead compare the reduced spectral densities, the similarity vanishes, since for slab geometries the geometrical weighting factor is $1 / \cos \psi$ (equation (B23)), rather than the $1 / \sin \psi$ appropriate for $2 \mathrm{D}$ turbulence. Figure 7 shows a comparison of the reduced spectral densities for 2D and slab fluctuations. The usual choice of parameters is in effect (standard bc's, $V_{A r 0} / U=1$ ), and for the slab solutions $k_{1}=0$. In this case the omnidirectional solutions are identical (solid curves), but the reduced forms show substantial differences (broken
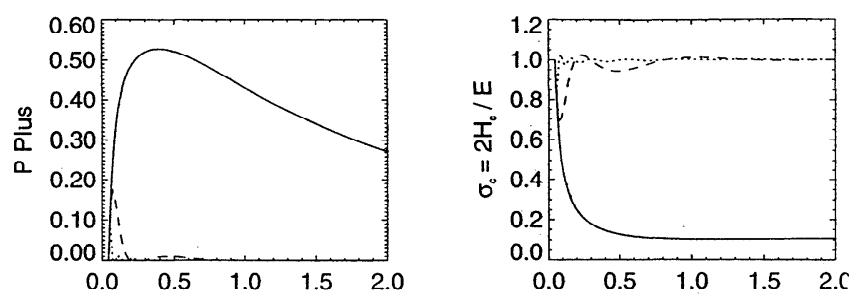

Figure 6. Plots showing the convergence of slab solutions toward WKB results as the wavenumber is increased (standard bc's, $V_{A r 0}=U$ ). Solid curves: $k=0$; Dashed curves: $k=2$; Dotted curves: $k=5$. A unit wavenumber in code units is equivalent to $2 \pi / R_{0}$ in physical units. This approach to WKB results has also been discussed by Heinemann and Olbert [1980] for linear toroidal waves. The horizontal coordinate is heliocentric distance in $\mathrm{AU}$.
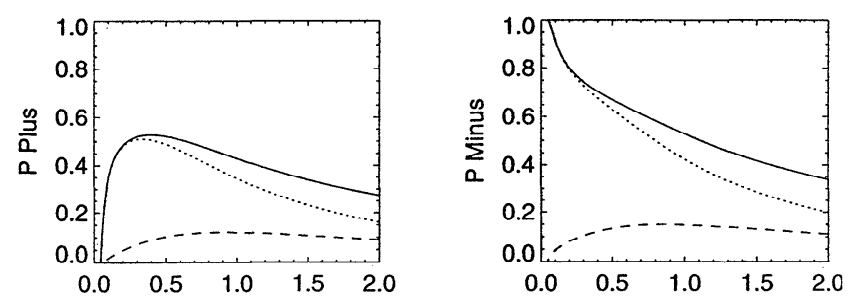

Figure 7. Comparison plots of the omnidirectional and reduced spectral amplitudes for $2 \mathrm{D}$ and slab turbulence. All plots are for standard bc's with $V_{A r 0}=U$. To avoid the oscillations seen in Figure 5, the slab curves are for $k_{1}=0$. Solid curves: omnidirectional spectral amplitudes for both 2D and slab fluctuations; Dashed curves: reduced spectral amplitudes for 2D fluctuations; Dotted curves: reduced spectral amplitudes for slab fluctuations. The reduced curves are calculated for $\alpha=5 / 3$. The horizontal coordinate is heliocentric distance in $\mathrm{AU}$.

curves). At smaller distances the reduced slab spectra differ little from their omnidirectional forms, since $\cos \psi \approx 1$ in this region. As $R$ increases, the geometrical factor leads to an increasing underemphasis of the power in the slab fluctuations. The situation is more or less reversed for $2 \mathrm{D}$ fluctuations, since here the geometrical factor involves $\sin \psi$. As noted above, normalized quantities are unaffected by reductions of this form.

\section{Comparison With Observations}

Figure 8 provides a summary of some of the lincar solutions presented above. Curves for the normalized cross helicity and the normalized energy difference, in the case where the turbulence is isotropic or 2D, are shown, as are several linear combinations of these solutions (all at a fixed but arbitrary inertial range wavenumber). Because the slab solutions depend so weakly on $k$ in the inertial range, it makes little difference which wavenumber is used here. The pure slab case
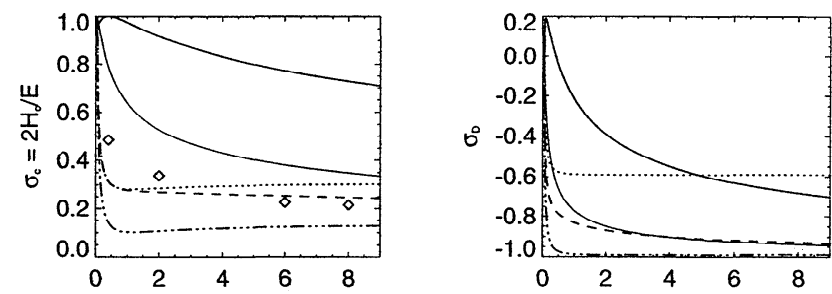

Figure 8. Plots of the numerical solutions to the linear transport equations for the normalized cross helicity and energy difference, with observational data points superimposed. The thick solid curves are for isotropic turbulence with $V_{A r 0} / U=1$, and the dot-dot-dotdash curves for $2 \mathrm{D}$ turbulence under the same conditions. The thin solid curve is the isotropic solution when $V_{A r 0} / U=0$ (analytic). Dotted curves: $80 \% 2 \mathrm{D}, 20 \%$ slab; Dashed curves: $60 \%$ isotropic $\left(V_{A r 0} / U=1\right), 40 \%$ $2 \mathrm{D}$. The diamonds denote observational data points obtained from three hour averages of Helios and Voyager data [Roberts et al., 1987a, b]. All solutions are computed from the omnidirectional spectra. The horizontal coordinate is heliocentric distance (AU). 
(not plotted) is very well approximated by the WKB result: $\sigma_{c}=1, \sigma_{D}=0$. Also shown are data points for $\sigma_{c}$ derived from three hour averages of Helios and Voyager data [Roberts et al., 1987a, b], which were used for earlier comparison with analytic and computed solutions [Zhou and Matthaeus, 1990a; Oughton and Matthaeus, 1992]. While data points for $\sigma_{D}$ are not shown, observational analyses indicate that this quantity is roughly constant in the outer heliosphere, with a value of about -0.3 [Goldstein et al., 1994].

It is clear that the inertial range slab (WKB) solutions and the 2D solutions give limits on behavior seen in the linear transport model, and provide bounds for what can be represented by physically reasonable linear superposition of these solutions. The WKB solution has no mixing at all, and energy equipartition at all heliocentric distances. The $2 \mathrm{D}$ solutions provide for rapid decrease of normalized cross helicity $\left(\sigma_{c}\right)$ to a level of $\approx 0.1$ by about $0.5 \mathrm{AU}$, while the total energy becomes almost purely magnetic by $1 \mathrm{AU}$. Clearly these effects of mixing are excessive, since they overshoot the observations substantially.

Linear combinations of $2 \mathrm{D}$ and slab solutions have values of $\sigma_{D}$ which are approximately constant beyond $1 \mathrm{AU}$ (e.g., the 80-20 mix shown in Figure 8). Choosing a $30 \% 2 \mathrm{D}, 70 \%$ slab mix, would allow matching of the theory and observations for this quantity; however, $\sigma_{c}$ for such a mixture is far too high to agree with observations. With a linear 2D-slab mixture, it is not possible to obtain simultaneously good (quantitative) agreement between theory and observations for both $\sigma_{c}$ and $\sigma_{D}$. However, models such as the $80 \% 2 \mathrm{D}, 20 \%$ slab mixture are both qualitatively reasonable and quantitatively better than any model with pure symmetry that we have tried. For example, this model has $\sigma_{c} \approx 0.3$ and $\sigma_{D} \approx-0.6$ at $1 \mathrm{AU}$.

Recently, Matthaeus et al. [1990] constructed a twodimensional correlation function for solar wind fluctuations using ISEE 3 data collected near $1 \mathrm{AU}$. As stated by Matthaeus et al. $[1990$, p. 20,678$]$, the simplest way to interpret this correlation function is to "assume that the solar wind at $1 \mathrm{AU}$ contains a population of Alfvénic (slablike) fluctuations, probably originating at the solar corona, along with a quasi-two-dimensional component that could be evidence of turbulent evolution between the sun and 1 AU." The difficulties associated with obtaining enough suitable data meant that only a single such correlation function was calculated, so that without further examples generalizations based on this single event must be handled with care. Nonetheless, an idealized model of the solar wind was postulated, viewing it as a fluid containing both slablike Alfvénic fluctuations and quasi-two-dimensional fluctuations. As (spectral) anisotropy seems to be a feature of turbulent evolution in the presence of a large-scale magnetic field [e.g., Shebalin et al., 1983; Hossain et al., 1985; Oughton et al., 1994a, b]-meaning that the fluctuations evolve toward a quasi-2D state-Matthaeus et al. [1990] predicted that quasi-two-dimensionality would become more important in the outer heliosphere. Fig- ure 8 shows that for (strong) $2 \mathrm{D}$ turbulence, $\sigma_{c}$ and $\sigma_{D}$ saturate at approximately constant values somewhere around a few AU. For ideal slab fluctuations, these normalized quantities are constant for all distances beyond $R_{0}$, so that our numerical solutions support the twocomponent interpretation just referred to.

\section{Discussion}

The numerical results presented here confirm that in valid regions of parameter space mixing terms induce substantial departures from leading order WKB solutions. Application of the non-WKB framework to the solar wind, using only the linear terms, has shown that simple mixing models can produce interesting levels of agreement with observations for quantities such as $\sigma_{c}$ and the small-scale Alfvén ratio, $r_{A}=E^{v} / E^{b}$. With the exception of the slab solutions, the curves for $\sigma_{c}$ are in qualitative agreement with the observations, showing significant decay with heliocentric distance. The quantitative agreement is certainly not good enough to support claims that mixing is the sole explanation for the observed decrease in $\sigma_{c}$; nonetheless, it does suggest that mixing, as computed here using the linear transport equations plays a role which, (1) cannot be neglected, and (2) is capable of explaining some of the evolutionary trends.

Previous results [Oughton and Matthaeus, 1992; Oughton, 1993; Tu and Marsch, 1993] of linear transport computations, as well as those given here, have suggested that the correspondence of linear solutions to observations can be made to agree better by using two component models which postulate some linear combination of fluctuation spectra with differing rotational symmetries. Of particular interest is that pure slab or pure isotropic turbulence is not at all a good fit to the observations. On the other hand, a mixture of slab and 2D fluctuations (about $80 \%$ 2D, 20\% slab, cf. Figure 8 ) is a reasonable fit to the observations. The latter mixture was also arrived at as a near optimum representation of observed (inferred) energetic particle mean free paths by predictions of dynamical, two component quasi-linear scattering theory [Bieber et al., 1994]. Other superpositions of transport solutions are reasonable fits as well, as is seen for example in th. $60 \%$ isotropic, $40 \% 2 \mathrm{D}$ model in Figure 8 , which is only a little worse fit than the $80-202 \mathrm{D} /$ slab model.

The solutions presented above are in excellent agreement with the earlier discussion concerning the influence of the mixing terms. Specifically, mixing plays an increasingly important role as either $\mathbf{V}_{A} / U$ or $\mathbf{k} \cdot \mathbf{V}_{A}$ approaches zero. Indeed, the impact of the mixing terms on the radial evolution of the physical quantities is crucially affected by the size of the WKB-enforcing factor, $\mathbf{k} \cdot \mathrm{V}_{A}$. Unless $F$ and $J$ are essentially uncoupled the solutions are very close to the leading order WKB ones, with almost all the energy being in the outward-type fluctuations. For slab fluctuations this is true even at wavenumbers much smaller than those characterizing the inertial range (except at very small $R$ ). The fact 
that $J$ is identically zero for isotropic and 2D turbulence, means that of the various cases considered here, only the slab geometries can show convergence towards WKB solutions.

The question arises as to the scale on which $\mathbf{k} \cdot \mathbf{V}_{A}$ is small. The natural terms to compare it to are the effective mixing operators. Denoting the angle between $\mathbf{k}$ and $\mathbf{V}_{A}$ by $\gamma(\mathbf{R})$, we have the following ratio:

$$
\frac{\mathbf{k} \cdot \mathbf{V}_{A}}{M_{2 D}^{+}}=\frac{2\left(V_{A r 0} / U\right) k \cos \gamma}{\cos ^{2} \psi+\frac{V_{A r 0}}{U R}\left(3 \cos ^{2} \psi-2\right)} \text {. }
$$

For $V_{A r 0} / U \leq 1$, the denominator is bounded above by 2 , and decreases with increasing $R$; we may therefore consider it to be at most an order unity quantity. For 2D fluctuations $\cos \gamma=0$, so that the numerator is inherently zero and mixing is always strong. For slab fluctuations, however, $\cos \gamma=1$, and the numerator increases without bound with $k$. If $V_{A r 0} / U \sim 1$, as is usually the case, the wavenumbers need only be $\gtrsim 10$ (in appropriate units), for WKB effects to nullify mixing. Such wavenumbers lie well below the inertial range, so that inertial range fluctuations are expected to be WKB in character when these conditions apply. The solutions presented provide numerical support for these arguments (see also Figure 6).

We now return to the analytic solutions mentioned in the results section. In particular, we attempt to clarify a misinterpretation of them. In their section 8, Tu and Marsch [1993] (TM93) compare their model with observations and with a "ZM" model [Zhou and Matthaeus, 1990a]. However, the version of the ZM model they employ is the very simplest case only (isotropic symmetry, $\left.V_{A} / U \rightarrow 0\right)$. The physical content of the ZM model is much broader than suggested by their comparison. Second, the same authors, in Appendix A of TM93 [p. 1273], develop a criticism of the ZM discussion of mixing. While they do not dispute ZM's conclusions, they prefer a description of the same effect based upon "convection of the fluctuation vorticity lines and fluctuation magnetic field lines." The mathematical expression of the conservation principle they use (in equations (A13)-(A14) of TM93) is, however, approximate and valid only in certain special cases, one of which is the $V_{A} / U \rightarrow 0$ limit. Their restatement of ZM's conclusion that kinetic and magnetic energy evolve independently in that limit is correct for $V_{A}=0$ only. As they also recognize, the mixing effect can occur in much wider circumstances so that we prefer our original interpretation. We also point out that their analytic solution (TM93, equations (A18)-(A22)) is developed from a set of $V_{A}=0$ equations for two-dimensional fluctuations (which TM93 elect to call convective structures), but they claim a comparison with ZM's result for onedimensional waves. Along similar lines, Appendix B of TM93 is devoted to a rederivation of the solutions to ZM's (97)-(99) (equations (B10)-(B11) in TM93). This $\mathrm{ZM}$ model is a $V_{A}=0$ three-dimensional isotropic steady solution with a power law inertial range. However, the framework used by TM93 begins with the convective structure equations (2D) and then invokes a $3 \mathrm{D}$ isotropic property of reduced spectra (TM93 equation (B8)) to arrive at the desired result. Because of these confusions, we have difficulty in evaluating the comparisons made by TM93.

All solutions of the linear transport model presented here have ignored the nonlinear effects that can enter the transport equations due to local MHD turbulence [Zhou and Matthaeus, 1990b; Tu and Marsch, 1990]. One problem in developing such a theory is the lack of precise knowledge of how to model the local turbulence effects associated with each spectral quantity. In fact, for certain spectral quantities, such as the energy difference $F(\mathbf{k})$, it is fair to say that this knowledge is very imprecise [Mangeney et al., 1991; Zhou and Matthaeus, 1990b]. In addition, as mentioned earlier, models with turbulent spectral transfer require $\mathbf{k}$ space boundary data in the inertial range, and an additional model, such as a recently proposed model for solar wind energy-containing eddies [Matthaeus et al., 1994a], to drive the inertial range transfer. Although such a complete model is still under development, a recent Ph.D. thesis [Oughton, 1993] contains preliminary results for models with energy-containing range, inertial range and modeled nonlinear effects. Although these computations are qualitatively similar to many of those shown here, the mixing effect appears to be much weaker in many cases. This can be attributed to the strong nonlinear term that was chosen to restore equipartition of magnetic and kinetic energy. Even though the origin of this term is somewhat ad hoc, a reasonable set of models for the nonlinear terms that permits strong mixing has yet to be arrived at. This has led to the tentative conclusion [Oughton, 1993] (see also Goldstein et al. [1994]) that mixing effects solely driven by expansion (i.e., spherically symmetric flows with a simple spiral magnetic field) can probably account for only a small part of the observed reduction in cross helicity in the solar wind. At present, turbulence driven by streamshear [Roberts et al., 1992] appears to be a strong candidate for explaining most of the reduction. In principle two scale transport equations can be developed that include nonspherically symmetric structures associated with streams; such models could be used to investigate the stream-shear driven model. In addition, we have suggested [Matthaeus et al., 1994a] that simple source terms can be added to the spherically symmetric transport models that can at least crudely model the effects of stream shear. At present, inclusion of shear effects and development of more accurate models of nonlinear effects would have to be considered at the forefront of needed improvements to the present solar wind transport models.

As a final point, we remark on some aspects of this work which pertain to the interpretation of the observational data. The complementary character of the geometrical effects for 2D and slab reduced spectra can introduce an apparent radial evolution of the turbulence, independent of any dynamics associated with the turbulence. To see this consider the following idealized example. Suppose that the interplanetary turbulence 
consists of two noninteracting components, namely 2D and slab fluctuations. Suppose also that for a given inertial range wavenumber, the omnidirectional spectral amplitudes of the two components are equal, i.e., $Q_{2 D}^{\text {omni }}(k)=Q_{\text {slab }}^{\text {omni }}(k)$, at all distances. Then, assuming that the omnidirectional spectra follow power laws $\left(k^{-\alpha}\right)$, and using the results of Appendix B, it follows that the ratio of the reduced spectral densities is given by

$$
\frac{Q_{2 D}^{\text {red }}\left(k_{1}\right)}{Q_{\text {slab }}^{\text {red }}\left(k_{1}\right)}=\frac{I(\alpha)}{\pi} \tan ^{\alpha-1} \psi .
$$

For $\alpha=1$, which is often observed at distances less than $0.5 \mathrm{AU}$, this ratio is constant and less than unity at all distances, indicating that the 2D fluctuations are de-emphasized relative to the true power levels in the $2 \mathrm{D}$ and slab components. When $\alpha=\frac{5}{3}$, the ratio is unity at $\psi \simeq 80^{\circ}$; at this point a crossover occurs from over-emphasis of the slab component to over-emphasis of the $2 \mathrm{D}$ portion. Observationally $\psi \approx 45^{\circ}$ at $1 \mathrm{AU}$, so that under these conditions the crossover occurs well into the outer heliosphere. Assuming that the 2D component has triple the power of the slab portion moves the crossover into about $1 \mathrm{AU}$. In any case, even if the turbulence undergoes no dynamical evolution (between two points on the same radial), the reduced spectra of unnormalized quantities will still show radial evolution. This point has also been discussed by Tu and Marsch $[1992,1993]$. Since for some time to come it is likely that only reduced spectra will be extractable from observational data, the problems associated with deconvolving geometrical and dynamical evolution effects will require further investigation. Fortunately, such problems do not necessarily arise for analytic and numerical work on the linear equations, since we always know what we started with. However, accurate representation of turbulence near the critical point seems to rule out use of the reduced spectral representation whenever there is a significant 2D component present. The reason is clear: the (radially) reduced spectra for such fluctuations vanish, even though finite energy is present in the spectra.

\section{Appendix A: Derivation of the Mixing Operators}

When referring to the large-scale fields it is convenient to work in heliocentric spherical polar coordinates $(R, \theta, \phi)$, where $R$ is the radial distance from the center of the sun, $\theta$ the co-latitude with respect to the solar rotation axis, and $\phi$ the azimuthal angle. The "1" coordinate corresponds to $R$, etc, so that, for example, the "1-3" component of the matrix form for $\nabla \mathbf{B}_{0}$ is given by evaluation of $\hat{\mathbf{R}}(\partial / \partial R)\left(\hat{\boldsymbol{\phi}} B_{0 \phi}\right)$. The approximations used for the large-scale fields may then be written

$$
\begin{aligned}
\rho & =\rho_{0}\left(\frac{R_{0}}{R}\right)^{2}, \\
\mathbf{U} & =U \hat{\mathbf{R}},
\end{aligned}
$$

$$
\begin{aligned}
& B_{0 r}=B_{0 r}^{0}\left(\frac{R_{0}}{R}\right)^{2} \\
& B_{0 \theta}=0 \\
& B_{0 \phi}=-B_{0 r}^{0} \frac{\Omega R_{0}}{U} \frac{R_{0}}{R}\left(1-\frac{R_{0}}{R}\right) \sin \theta
\end{aligned}
$$

where $R_{0}, \rho_{0}, U, B_{0 r}^{0}$, and $\Omega$ (the solar rotation rate), are constants. However, for generality, in this appendix we redefine $B_{0 r}^{0}$ to be $B_{0 r}^{0} f(\theta)$. Choosing $f(\theta)=\cos \theta$, for example, introduces a planar current sheet at the heliocentric equator.

Substituting the above forms into the defining equation for the mixing tensors (4) it is straightforward to arrive at the following matrix forms

$$
\begin{aligned}
M_{j i}^{ \pm}= & \left(\begin{array}{ccc}
-1 & 0 & 0 \\
0 & 1 & 0 \\
0 & 0 & 1
\end{array}\right) \frac{U}{2 R} \pm\left(\begin{array}{ccc}
-5 & 0 & 0 \\
2 f^{\prime} / f & 1 & 0 \\
0 & 0 & 1
\end{array}\right) \frac{V_{A r}}{2 R} \\
& \pm\left(\begin{array}{ccc}
0 & 0 & -\frac{R-2 R_{0}}{R-R_{0}} \\
0 & 0 & \cot \theta+f^{\prime} / f \\
-1 & -\cot \theta & 0
\end{array}\right) \frac{V_{A \phi}}{R}
\end{aligned}
$$

where $f^{\prime}=d f / d \theta$. Zhou and Matthaeus [1990a] performed this calculation for the $\mathbf{B}_{0}=0$ case, which is relevant when $V_{A} / U \ll 1$, as in the outer heliosphere for example. Note that only the first of these matrices is index symmetric.

The mixing tensors never appear in isolation, but only contracted, on either one or both indices, with a small-scale tensor field (the Einstein summation convention is in effect). Denoting such a small-scale field by $Q_{j i}$, the most general form of the contraction which we need to consider is $Q_{j \alpha} M_{\alpha i}^{ \pm}$, the full contraction obviously being obtained by setting $i=j$. In order to calculate these contractions we need to know something regarding the form of $Q_{i j}$. Imposing particular symmetry properties on the turbulence provides this information, at least in the three cases we consider here: turbulence which is isotropic, $2 \mathrm{D}$, or slab. We now show how to perform the appropriate contractions for inertial range fluctuations.

When representing the small-scale fluctuations we are free to work in any coordinate system we desire, the small-scale coordinates ( $\mathbf{x}$ and $\mathbf{r}$ ) being considered independent of the large-scale coordinate (R). A sensible choice, and the one we adopt, is to work in a Cartesian system which is locally aligned with the large-scale heliocentric coordinate system just discussed. Denoting the unit vectors of this system by $\mathbf{e}_{1}, \mathbf{e}_{2}$, and $\mathbf{e}_{3}$, we are therefore insisting on the following relationships:

$$
\begin{array}{llll}
\mathbf{e}_{1} & \| & \hat{\mathbf{R}} \\
\mathbf{e}_{2} & \| & \hat{\theta} \\
\mathbf{e}_{3} & \| & \hat{\phi}
\end{array}
$$

For $2 \mathrm{D}$ and slab fluctuations it will also be convenient to work with a small-scale coordinate system which has 
the "1" direction aligned with the local value of $\mathbf{B}_{0}$. We denote quantities associated with this coordinate system using primes ('), with unit vectors $\mathbf{e}_{1}^{\prime}, \mathbf{e}_{2}^{\prime}$, and $\mathbf{e}_{3}^{\prime}$. In order to make transforming between the radially (unprimed) and field (primed) aligned systems as simple as possible, we choose $\mathbf{e}_{2}^{\prime}=\mathbf{e}_{2}$, so that $\mathbf{B}_{0}$ lies in the $\mathbf{e}_{1^{-}}$ $\mathbf{e}_{3}$ plane. The transformation is then simply a rotation about $\mathbf{e}_{2}=\hat{\theta}$ by $-\psi$. It follows that to transform a second rank tensor in the field-aligned system, say $\mathrm{T}^{\prime}$, to one in the radially aligned system, say $T$, we need only apply the following rule:

$$
\mathrm{T}=\mathrm{OT}^{\prime} \mathrm{O}^{T}
$$

where superscript $T$ indicates the transpose operation, and

$$
O=\left(\begin{array}{ccc}
\cos \psi & 0 & \sin \psi \\
0 & 1 & 0 \\
-\sin \psi & 0 & \cos \psi
\end{array}\right)
$$

is the appropriate rotation matrix.

Consider first the (nonhelical) isotropic case. Theory and experiment suggest that the modal spectrum of inertial range fluctuations exhibits a power law dependence on the wavenumber k [e.g., Kolmogorov, 1941a; Kraichnan, 1965; Grant et al., 1962]. Denote the appropriate modal spectrum by

$$
\Sigma_{i j}(\mathbf{k})=\frac{Q^{\mathrm{omni}}(k)}{8 \pi k^{2}}\left(\delta_{i j}-\frac{k_{i} k_{j}}{k^{2}}\right),
$$

where

$$
Q^{\mathrm{omni}}(k)=\int \Sigma_{\beta \beta}(\mathbf{k}) d \mathbf{A}(\mathbf{k})
$$

is the average of $\Sigma_{\beta \beta}(\mathbf{k})$ over all wave vectors $\mathbf{k}$ of the same magnitude $k$. It can also be shown that the full omnidirectional spectrum takes the form

$$
\begin{aligned}
Q_{i j}^{\mathrm{omni}}(k) & =\int \Sigma_{i j}(\mathbf{k}) d \mathbf{A}(\mathbf{k}) \\
& =\frac{1}{3} \delta_{i j} Q^{\mathrm{omni}}(k) .
\end{aligned}
$$

We can now evaluate the contraction of an isotropic omnidirectional spectrum with the mixing tensors to obtain

$$
\begin{aligned}
Q_{\alpha \beta}^{\mathrm{omni}} M_{\beta \alpha}^{ \pm} & =\frac{1}{2 R}\left[\frac{U}{3} \mp V_{A r}\right] Q^{\mathrm{omni}}(k) \\
& =M_{\mathrm{iso}}^{ \pm} Q^{\mathrm{omni}}(k),
\end{aligned}
$$

where we refer to the scalar quantities $M_{\text {iso }}^{ \pm}$as the effective mixing operators for the omnidirectional spectra of (nonhelical) isotropic turbulence. The subscript "iso" will often be dropped, since the type of turbulence being considered is usually clear from the context. Note the sign change in $M^{+}$at $R=3 R_{0}\left(V_{A r} \propto R_{0} / R\right)$.
The diagonal character of $Q_{i j}^{\text {omni }}$ has precluded the picking up of any of the matrix terms in $M_{j i}^{ \pm}$which involve $\theta$. As it turns out, this is also true for $2 \mathrm{D}$ and slab turbulence (apart from the $\theta$ dependence of $V_{A \phi}$ ). Thus any $\theta$ dependence of $B_{0 r}$, i.e., $f(\theta) \neq$ const, contributes to the mixing operators only through its inclusion in $V_{A r}$ and/or $V_{A \psi}$; terms in $f^{\prime}$ certainly appear in $M_{j i}^{ \pm}$, but they are missed when the full contraction with $Q_{i j}^{\text {omni }}$ is calculated. It is also evident that the $M_{\text {iso }}^{ \pm}$ operators do not depend on $V_{A \phi}$, and, in fact, such dependence will be lacking for all $Q_{i j}$ which are diagonal in the radially aligned coordinate system.

For 2D turbulence it can be shown that in the fieldaligned coordinate system $Q_{i j}^{\text {omni }}$ takes the form

$$
Q_{i j}^{\mathrm{omni}}\left(k_{\perp}\right)=\frac{1}{2}\left(\begin{array}{ccc}
0 & 0 & 0 \\
0 & 1 & 0 \\
0 & 0 & 1
\end{array}\right) Q^{\mathrm{omni}}\left(k_{\perp}\right) \delta\left(k_{\|}\right),
$$

where $k_{\perp}$ is the magnitude of the projection of the wave vector into the $2 \mathrm{D}$ plane perpendicular to $\mathbf{B}_{0}$, and $k_{\|}$is the component along $\mathbf{B}_{0}$. The presence of the $\delta$ function forces the equivalence of $\mathbf{k}_{\perp}$ and $\mathbf{k}$. Making use of (A7) to transform this to the radially aligned system and then contracting with the mixing tensors, we obtain

$$
Q_{\alpha \beta}^{\mathrm{omni}} M_{\beta \alpha}^{ \pm}=M_{2 D}^{ \pm} Q^{\mathrm{omni}}\left(k_{\perp}\right) .
$$

where

$$
\begin{aligned}
M_{2 D}^{ \pm}= & \frac{1}{2 R}\left[U \cos ^{2} \psi \pm V_{A r}\left(3 \cos ^{2} \psi-2\right)\right. \\
& \left. \pm \frac{1}{2} V_{A r 0}\left(\frac{\Omega R_{0}}{U}\right)\left(2-\frac{3 R_{0}}{R}\right) \sin \theta \sin 2 \psi\right]
\end{aligned}
$$

For slab turbulence the wave vectors (magnitude $k_{\|} \equiv$ $k$ ) are parallel to the mean magnetic field $\mathbf{B}_{0}$, with all the power being in directions perpendicular to $\mathbf{B}_{0}$. If we further assume that the power is distributed isotropically over these directions, then, in the field-aligned coordinate system, the omnidirectional spectra are of the form

$$
Q_{j k}^{\mathrm{omni}}\left(k_{\|}\right)=\frac{1}{2}\left(\begin{array}{ccc}
0 & 0 & 0 \\
0 & 1 & 0 \\
0 & 0 & 1
\end{array}\right) Q^{\mathrm{omni}}\left(k_{\|}\right) \delta\left(k_{\perp}\right),
$$

The formal similarity to the $2 \mathrm{D}$ case is evident so that we immediately have $M_{\text {slab }}^{ \pm}=M_{2 D}^{ \pm}$. In the limit that $\psi(\mathbf{R}) \rightarrow 0$, these operators become those applicable for slab waves which propagate parallel to the radial direction.

Finally, we note that Tu and Marsch [1993] have recently discussed the nature of the mixing tensors when $\mathbf{V}_{A}=0$. In this limit $M^{+}=M^{-}$, but, as they point out, in this case the Elsässer variable representation actually obscures the physics somewhat, since there is no $\mathbf{B}_{0}$. Considering just the linear terms in the transport equations for the (traced) spectra of the (small-scale) kinetic energy, magnetic energy, and cross helicity (cf. those for $P^{ \pm}$and $F$ ), it becomes clear that these equa- 
tions are independent, i.e., no coupling amongst $E^{v}$, $E^{b}, H_{c}$ occurs (see Appendix B of their paper). As the authors stated, the basic physics then consists of advection and expansion of the vorticity and magnetic field fluctuations. While it is still true that the Elsässer fluctuations are mixed, Tu and Marsch's point, that in the absence of a large-scale magnetic field (and nonlinearities), the velocity and magnetic field fluctuations decouple, is well taken and serves as a reminder regarding the interpretation of the Elsässer variables for such situations.

\section{Appendix B: The Relationship Between Reduced and Omnidirectional Spectra}

For the purposes of comparison between theory and observations it is useful to know how to obtain reduced spectra from the omnidirectional forms. We now show how to do this, under certain assumptions, for the three types of fluctuations considered in this paper. Notation and results from Appendix $A$ are made use of.

For 3D isotropic turbulence the tensor form of the reduced spectrum (reduced along the radial direction $\mathbf{e}_{1}$, radial wavenumber $k_{1}$ ) is defined by

$$
Q_{i j}^{\mathrm{red}}\left(k_{1}\right)=\int d k_{2} d k_{3} \Sigma_{i j}(\mathbf{k}),
$$

so that the traced form is

$$
\begin{aligned}
Q^{\mathrm{red}}\left(k_{1}\right) & =Q_{\beta \beta}^{\mathrm{red}}\left(k_{1}\right) \\
& \equiv \frac{1}{2} \int_{k_{1}}^{\infty} d k \frac{Q^{\mathrm{omni}}(k)}{k}
\end{aligned}
$$

[e.g., Batchelor, 1970; Zhou and Matthaeus, 1990a]. If $Q^{\text {omni }}(k) \propto k^{-\alpha}$, then $Q^{\text {red }}\left(k_{1}\right)$ exhibits the same power law dependence. Specifically,

$$
Q^{\text {red }}\left(k_{1}\right)=\frac{1}{2 \alpha} Q^{\text {omni }}\left(k_{1}\right) .
$$

Important values of $\alpha$ include those associated with the theories of Kolmogorov $(\alpha=5 / 3)$ and Kraichnan $(\alpha=$ $3 / 2$ ).

For 2D turbulence the relationship between $Q_{\beta \beta}^{\text {omn }}$ and $Q_{\beta \beta}^{\text {red is }}$

$$
\begin{aligned}
Q^{\mathrm{red}}\left(k_{1}\right) & =\int_{-\infty}^{\infty} d k_{2} \frac{Q^{\mathrm{omni}}\left(k_{\perp}\right)}{2 \pi k_{\perp}} \\
& =\frac{1}{\pi \sin \psi} \int_{\frac{k_{1}}{\sin \psi}}^{\infty} \frac{Q^{\mathrm{omni}}(x) d x}{\sqrt{x^{2}-k_{1}^{2} / \sin ^{2} \psi}}
\end{aligned}
$$

where the change of variables $x^{2}=k_{2}^{2}+k_{1}^{2} / \sin ^{2} \psi=k_{\perp}^{2}$ has been used. When $\psi=0$ the expression (B20) is singular and a slightly different form holds (not shown). In essence, when $\psi=0$, we have $Q^{\text {red }}\left(k_{1}\right)=0$ for $k_{1}>0$ because $2 \mathrm{D}$ fluctuations do not vary along $\mathbf{B}_{0}$ which is radial in this case. The quantity $k_{1} / \sin \psi$ may be interpreted as the magnitude of the projection of $\mathbf{k}_{\perp}$ onto the $\mathbf{e}_{1}^{\prime}-\mathbf{e}_{3}^{\prime}$ plane, or more specifically onto the $\mathbf{e}_{3}^{\prime}$ axis, since $\mathbf{k}_{\perp}$ lies in the $\mathbf{e}_{2}-\mathbf{e}_{3}^{\prime}$ plane. A related perspective is that it corresponds to the component of $\mathbf{k}_{\perp}$ in the plane of $\mathbf{B}_{0}$.

Assuming again a power law omnidirectional spectrum, $Q^{\text {omni }}(x) \propto x^{-\alpha}$, and using the variable substitution $\sqrt{t}=k_{1} /(x \sin \psi)$, yields

$$
Q^{\mathrm{rcd}}\left(k_{1}\right)=\frac{I(\alpha)}{\pi \sin \psi} Q^{\mathrm{omni}}\left(\frac{k_{1}}{\sin \psi}\right)
$$

where

$$
\begin{aligned}
I(\alpha) & =\frac{1}{2} \int_{0}^{1} t^{\frac{1}{2}-1}(t-1)^{\frac{\alpha}{2}-1} d t \\
& =\frac{1}{2} \frac{\Gamma\left(\frac{1}{2}\right) \Gamma\left(\frac{\alpha}{2}\right)}{\Gamma\left(\frac{\alpha+1}{2}\right)}
\end{aligned}
$$

and $\Gamma$ denotes the gamma (factorial) function. The following values of $I(\alpha)$ are of particular interest $I(1)=$ $\pi / 2, I\left(\frac{3}{2}\right) \simeq 1.1982, I\left(\frac{5}{3}\right) \simeq 1.1203$, and $I(2)=1$.

For slab turbulence, the relation between the (traced) omnidirectional spectra and the reduced spectra is

$$
Q^{\mathrm{red}}\left(k_{1}\right)=\frac{1}{\cos \psi} Q^{\mathrm{omni}}\left(\frac{k_{1}}{\cos \psi}\right)
$$

The quantity $k_{1} / \cos \psi=|\mathbf{k}|=k_{\|}$is clearly the magnitude of the full wave vector.

Acknowledgments. Helpful discussions with Gary Zank, Duane Pontius, and Aaron Roberts are acknowledged. We thank Aaron Roberts for providing the observational data points and the referees for useful comments. This research has been supported in part by the NASA SPTP at Bartol, NSF grant ATM 9318728, and the UK PPARC at St Andrews. Computational resources were provided by the San Diego Supercomputing Center. Some of this research was previously published in a Ph.D. thesis submitted to the University of Delaware by S. Oughton

The Editor thanks A. Mangeney and E. Marsch for their assistance in evaluating this paper.

\section{References}

Barnes, A., Hydromagnetic waves and turbulence in the solar wind, in Solar System Plasma Physics, vol. I, edited by E. N. Parker, C. F. Kennel, and L. J. Lanzerotti, p. 251, North-Holland, New York, 1979.

Batchelor, G. K., The Theory of Homogeneous Turbulence, Cambridge University Press, New York, 1970.

Bavassano, B., M. Dobrowolny, F. Mariani, and N. F. Ness, Radial evolution of power spectra of interplanetary Alfvénic turbulence, J. Geophys. Res., 87, 3617, 1982a.

Bavassano, B., M. Dobrowolny, G. Fanfoni, F. Mariani, and N. F. Ness, Statistical properties of MHD fluctuations associated with high-speed streams from Helios-2 observations, Sol. Phys., 78, 373, $1982 \mathrm{~b}$.

Belcher, J. W., and L. Davis, Large-amplitude Alfvén waves in the interplanetary medium, 2, J. Geophys. Res., 76, 3534, 1971.

Bieber, J. W., W. H. Matthaeus, C. W. Smith, W. Wanner, M. Kallenrode, and G. Wibberenz, Proton and electron 
mean free paths: The Palmer consensus revisited, Astrophys. J., 420, 294, 1994.

Canuto, C., M. Y. Hussaini, A. Quarteroni, and T. A. Zang, Spectral Methods in Fluid Mechanics, Springer Ser. Comput. Phy., Springer-Verlag, New York, 1988.

Goldstein, M. L., D. A. Roberts, and W. H. Matthaeus, Magnetohydrodynamic turbulence in cosmic winds, in Cosmic Winds and the Heliosphere, edited by J. R. Jokipii, C. P. Sonnett, and M. S. Giampapa, University of Arizona Press, Tucson, 1994.

Gottlieb, D., and S. A. Orszag, Numerical Analysis of Spectral Methods: Theory and Applications, Society for Industrial and Applied Mathematics, Philadelphia, 1977.

Grant, H. L., R. W. Stewart, and A. Moilliet, Turbulence spectra from a tidal channel, J. Fluid Mech., 12, 241, 1962.

Heinemann, M., and S. Olbert, Non-WKB Alfvén waves in the solar wind, J. Geophys. Res., 85, 1311, 1980.

Hollweg, J. V., Alfvén waves in a two-fluid model of the solar wind, Astrophys. J., 181, 547, 1973a.

Hollweg, J. V., Alfvén waves in the solar wind: Wave pressure, Poynting flux, and angular momentum, J. Geophys. Res., 78, 3643, 1973b.

Hollweg, J. V., Transverse Alfvén waves in the solar wind: Arbitrary k, $\mathbf{v}_{0}, \mathbf{B}_{0}$, and $\delta b, J$. Geophys. Res., 79, 1539, 1974.

Hollweg, J. V., On WKB expansions for Alfvén waves in the solar wind, J. Geophys. Res., 95, 14,873, 1990.

Hossain, M., G. Vahala, and D. Montgomery, Forced magnetohydrodynamic turbulence in a uniform magnetic field, Phys. Fluids, 28, 3074, 1985.

Hundhausen, A. J., Coronal Expansion and the Solar Wind, Springer-Verlag, New York, 1972.

Kolmogorov, A. N., Local structure of turbulence in an incompressible viscous fluid at very high Reynolds numbers, C.R. Acad. Sci. U.R.S.S., 30, 301, 1941.

Kraichnan, R. H., Inertial-range spectrum of hydromagnetic turbulence, Phys. Fluids, 8, 1385, 1965.

Lotova, N. A., The solar wind transsonic region, Solar Phys., 117, 399, 1988.

Lotova, N. A., D. F. Blums, and K. V. Vladimirskii, Interplanetary scintillation and the structure of the solar wind transonic region, Astron. Astrophys., 150, 266, 1985.

Mangeney, A., R. Grappin, and M. Velli, MHD turbulence in the solar wind, in Advances in Solar System Magnetohydrodynamics, edited by E. R. Priest and A. W. Hood, p. 327, Cambridge University Press, New York, 1991.

Marsch, E., and A. Mangeney, Ideal MHD equations in terms of compressive Elsässer variables, J. Geophys. Res., 92, 7363, 1987.

Marsch, E., and K. Richter, Distribution of solar wind angular momentum between particles and magnetic field: Inferences about the Alfvén critical point from the Helios observations, J. Geophys. Res., 89, 5386, 1984.

Marsch, E., and C.-Y. Tu, Dynamics of correlation functions with Elsässer variables for inhomogeneous MHD turbulence, J. Plasma Phys., 41, 479, 1989.

Marsch, E., and C.-Y. Tu, Spectral and spatial evolution of compressible turbulence in the inner solar wind, $J$. Geophys. Res., 95, 11945, 1990.

Marsch, E., and and C.-Y. Tu, Modeling results on spatial transport and spectral transfer of solar wind Alfvénic turbulence, J. Geophys. Res., 98, 21 045, 1993.

Matthaeus, W. H., and M. L. Goldstein, Measurement of the rugged invariants of magnetohydrodynamic turbulence in the solar wind, J. Geophys. Res., 87, 6011, 1982a.

Matthaeus, W. H., and M. L. Goldstein, Stationarity of magnetohydrodynamic fluctuations in the solar wind, $J$. Geophys. Res., 87, 10347,1982 b.
Matthaeus, W. H., M. L. Goldstein, and D. A. Roberts, Evidence for the presence of quasi-two-dimensional nearly incompressible fluctuations in the solar wind, J. Geophys. Res., 95, $20673,1990$.

Matthaeus, W. H., M. L. Goldstein, and C. W. Smith, Evaluation of magnetic helicity in homogeneous turbulence, Phys. Rev. Lett., 48, 1256, 1982.

Matthaeus, W. H., S. Oughton, D. H. Pontius, Jr., and Y. Zhou, Evolution of energy containing eddies, J. Geophys. Res., 99, 19267,1994 a.

Matthaeus, W. H., Y. Zhou, G. P. Zank, and S. Oughton, Transport theory and the WKB approximation for interplanetary MHD fluctuations, J. Geophys. Res., 99, 23421 , $1994 \mathrm{~b}$.

Matthaeus, W. H., and Y. Zhou, Nearly incompressible MHD turbulence in the solar wind, in Nonlinear Phenomena in MHD Turbulence, edited by A. Pouquet, M. Meneguzzi, and P. L. Sulem, p. 93, Elsevier Science, North Holland, New York, 1989.

Matthaeus, W. H., Y. Zhou, S. Oughton, and G. P. Zank, Weakly inhomogeneous MHD turbulence and transport of solar wind fluctuations, in Proceedings of Solar Wind 7, COSPAR Colloq. Ser., vol. 3, edited by E. Marsch and R. Schwenn, p. 511, Pergamon, New York, 1992.

Mullan, D. J., Sources of the solar wind: What are the smallest-scale structures? Astron. and Astro., 232, 520, 1990.

Oughton, S., Transport of Solar Wind Fluctuations: A Turbulence Approach, Ph.D. thesis, Univ. of Del., Newark, 1993.

Oughton, S., and W. H. Matthaeus, Evolution of solar wind fluctuations and the influence of turbulent "mixing," in Proceedings of Solar Wind 7, COSPAR Colloq. Ser., vol. 3, edited by E. Marsch and R. Schwenn, p. 523, Pergamon, New York,1992.

Oughton, S., W. H. Matthaeus, and E. R. Priest, 3D MHD turbulence with a mean magnetic field, in Current Topics in Astrophysical and Fusion Plasma Research, edited by M. Heyn, W. Kernbichler, and H. K. Biernat, p. 81, Springer-Verlag, New York, 1994a.

Oughton, S., E. R. Priest, and W. H. Matthaeus, The influence of a mean magnetic field on three-dimensional MHD turbulence, J. Fluid Mech., 280, 95, 1994b.

Parker, E. N., Dynamics of the interplanetary gas and magnetic fields, Astrophys. J., 123, 644, 1958.

Parker, E. N., Dynamical theory of the solar wind, Space Sci. Rev., 4, 666, 1965.

Pizzo, V., R. Schwenn, E. Marsch, H. Rosenbauer, K.H. Mühlhäuser, and F. M. Neubauer, Determination of the solar wind angular momentum flux from the Helios data-An observational test of the Weber and Davis theory, Astrophys. J., 271, 335, 1983.

Roberts, D. A., M. L. Goldstein, and L. W. Klein, The amplitudes of interplanetary fluctuations: Stream structure, heliocentric distance, and frequency dependence, $J$. Geophys. Res., 95, 4203, 1990.

Roberts, D. A., M. L. Goldstein, W. H. Matthaeus, and S. Ghosh, Velocity shear generation of solar-wind turbulence, J. Geophys. Res., 97, 17 115, 1992.

Roberts, D. A., L. W. Klein, M. L. Goldstein, and W. H. Matthaeus, The nature and evolution of magnetohydrodynamic fluctuations in the solar wind: Voyager observations, J. Geophys. Res., 92, 11 021, 1987a.

Roberts, D. A., M. L. Goldstein, L. W. Klein, and W. H. Matthaeus, Origin and evolution of fluctuations in the solar wind: Helios observations and Helios-Voyager comparisons, J. Geophys. Res., 92, 12 023, $1987 \mathrm{~b}$.

Shebalin, J. V., W. H. Matthaeus, and D. Montgomery, Anisotropy in MHD turbulence due to a mean magnetic field, J. Plasma Phys., 29, 525, 1983. 
Tennekes, H., and J. L. Lumley, A First Course in Turbulence, MIT Press, Cambridge, Mass., 1972.

Tu, C.-Y., The damping of interplanetary Alfvenic fluctuations and the heating of the solar wind, J. Geophys. Res., 93, 7, 1988.

Tu, C.-Y., and E. Marsch, Transfer equations for spectral densities of inhomogeneous MHD turbulence, J. Plasma Phys., 44, 103, 1990.

Tu, C.-Y., and E. Marsch, The evolution of MHD turbulence in the solar wind, in Proceedings of Solar Wind 7, COSPAR Colloq. Ser., vol. 3, edited by E. Marsch and R. Schwenn, p. 549, Pergamon, New York, 1992.

Tu, C.-Y., and E. Marsch, A model of solar wind fluctuations with two components: Alfvén waves and convective structures, J. Geophys. Res., 98, 1257, 1993.

Tu, C.-Y., E. Marsch, and K. M. Thieme, Basic properties of solar wind MHD turbulence near 0.3 au analyzed by means of Elsässer variables, J. Geophys. Res., 94, 11739 , 1989.

Tu, C.-Y., Z.-Y. Pu, and F.-S. Wei, The power spectrum of interplanetary Alfvénic fluctuations: Derivation of the governing equation and its solution, J. Geophys. Res., 89, 9695, 1984.

Weber, E. J., and L. Davis, The angular momentum of the solar wind, Astrophys. J., 148, 217, 1967.

Zank, G. P., and W. H. Matthaeus, The equations of reduced magnetohydrodynamics, J. Plasma Phys., 48, 85, 1992a.
Zank, G. P., and W. H. Matthaeus, Waves and turbulence in the solar wind, J. Geophys. Res., 97, $17189,1992 \mathrm{~b}$.

Zank, G. P., and W. H. Matthaeus, Nearly incompressible fluids, II, Magnetohydrodynamics, turbulence, and waves, Phys. Fluids A, 5, 257, 1993.

Zhou, Y., and W. H. Matthaeus, Non-WKB evolution of solar wind fluctuations: A turbulence modeling approach, Geophys. Res. Lett., 16, 755, 1989.

Zhou, Y., and W. H. Matthaeus, Transport and turbulence modeling of solar wind fluctuations, J. Geophys. Res., 95, 10291,1990 a.

Zhou, Y., and W. H. Matthaeus, Remarks on transport theories of interplanetary fluctuations, J. Geophys. Res., $95,14863,1990 \mathrm{~b}$.

Zhou, Y., and W. H. Matthaeus, Models of inertial range spectra of interplanetary magnetohydrodynamic turbulence, J. Geophys. Res., 95, 14881,1990 c.

S. Oughton, Department of Mathematics, University College London, WC1E 6BT, England. (e-mail: sean@math.ucl.ac.uk)

W. H. Matthaeus, Bartol Research Institute, University of Delaware, Newark, DE 19716, USA. (e-mail: yswhm@brisvr.bartol.udel.edu)

(Received September 7, 1994; revised January 10, 1995; accepted February 17, 1995.) 